\title{
Complex dynamics induced by strong confinement - From tracer diffusion in strongly heterogeneous media to glassy relaxation of dense fluids in narrow slits
}

\author{
Suvendu Mandal ${ }^{1}$, Markus Spanner-Denzer ${ }^{2}$, Sebastian Leitmann ${ }^{1}$, and \\ Thomas Franosch ${ }^{1, a}$ \\ 1 Institut für Theoretische Physik, Universität Innsbruck, Technikerstr. 21A, \\ 6020 Innsbruck, Austria \\ 2 Institut für Theoretische Physik, Friedrich-Alexander-Universität Erlangen-Nürnberg, \\ Staudtstraße 7, 91058 Erlangen, Germany
}

Received 13 April 2017 / Received in final form 11 May 2017

Published online 10 August 2017

\begin{abstract}
We provide an overview of recent advances of the complex dynamics of particles in strong confinements. The first paradigm is the Lorentz model where tracers explore a quenched disordered host structure. Such systems naturally occur as limiting cases of binary glassforming systems if the dynamics of one component is much faster than the other. For a certain critical density of the host structure the tracers undergo a localization transition which constitutes a critical phenomenon. A series of predictions in the vicinity of the transition have been elaborated and tested versus computer simulations. Analytical progress is achieved for small obstacle densities. The second paradigm is a dense strongly interacting liquid confined to a narrow slab. Then the glass transition depends nonmonotonically on the separation of the plates due to an interplay of local packing and layering. Very small slab widths allow to address certain features of the statics and dynamics analytically.
\end{abstract}

\section{Introduction}

The dynamics of dense glass-forming liquids slows down drastically upon decreasing the temperature or compressing the system eventually reaching a quasi-arrested disordered structure referred to as a glass. Concomitant with the vitrification the slow structural relaxation displays a series of striking features which do not have an analog in other domains of physics [1]. In recent years significant progress has been achieved to provide accurate data covering the microscopic evolution of the structural relaxation from a few picoseconds up to the microsecond regime. While there is still no

\footnotetext{
${ }^{\mathrm{a}}$ e-mail: thomas.franosch@uibk.ac.at
} 
consensus on how the glass transition should be rationalized theoretically, a coherent theoretical picture encompassing various facets accompanying the glass transition has been developed within the mode-coupling theory (MCT) of the glass transition $[1,2]$ by Wolfgang Götze and collaborators in the last 30 years.

Various predictions of the MCT have been corroborated in experiments and simulations [3]. For example, strong evidence for the predicted minimum in the frequency-dependent susceptibilities has been collected in depolarized-lightscattering experiments [4-6] which have been analyzed in terms of schematic models within the MCT [7-9]. The freezing of the spatio-temporal dynamics accompanied by stretched relaxation has been directly observed in dynamic light scattering in colloidal systems $[10,11]$ which strikingly resembles the prediction of MCT $[12,13]$ for hard spheres. Seminal computer simulations on binary mixtures $[14,15]$ have observed scaling behavior for the relaxation in the close vicinity of a plateau in the intermediate scattering functions characterizing the frozen-in structure. A highly non-trivial prediction of the MCT is the existence of glass-glass transitions if competing mechanisms for glass formation exist $[16,17]$. For polymer-colloid mixtures an additional short-range depletion attraction can be tuned such that the nonequilibrium state diagram displays nonmonotonic glasstransition lines [18-22], i.e. a reentrant behavior emerges. Then for certain size ranges of the depletion interaction a higher-order glass transition singularity becomes manifest in the dynamics accompanied by ultra-slow logarithmic decays $[16,17,23-25]$.

The theoretical progress in understanding the complex dynamics accompanied with the glass transition encourages now to push mode-coupling theory to new frontiers. So far MCT calculations were restricted to the equilibrium dynamics for monocomponent or binary systems of comparable size. Here we now focus on systems where the constituents are only weakly coupled and one component diffuses much faster than the other one. Such a decoupling of the dynamics has been observed in alkali silica melts in computer simulations $[26,27]$ and in neutron scattering experiments $[28,29]$. Microscopically the alkali ions meander through a slowly rearranging host matrix of silicon oxide on preferential diffusion pathways, reminiscent of a nano-porous material. Semi-quantitative agreement with MCT has been achieved [30] using the measured static structure factors as input in the mode-coupling functional. Yet, at first glance silica melts appear as a peculiarity since they are known to form locally tetrahedral networks, i.e. with strong directional bonds yielding only a loosely packed host structure. However, computer simulations for strongly size-disparate mixtures interacting via soft-sphere repulsion [31,32] or repulsive Yukawa interactions [33] display the same phenomenology without invoking chemical effects or polymeric aspects [34]. Therefore one infers that the scenario of weakly coupled dynamics is generic. Within MCT this is connected to a delocalization transition for the small particles [35] which diffuse through an arrested matrix of big particles. An experimental realization of such a strongly size-disparate mixture has been achieved for colloids only recently by Sentjabrskaja et al. [36]. There the experimental challenge was to resolve the dynamics of the small particles which rearrange too fast for conventional particle tracking. By adapting differential dynamics microscopy also to dense colloidal systems they were able to extract intermediate scattering functions for the small particles and uncover a novel ultra-slow relaxation scenario for a certain critical size ratio.

The limiting case where one component is frozen from the very beginning has been addressed theoretically in terms of an extension of MCT by Krakoviack [37-40]. Here an interacting liquid is confined to a quenched porous host structure. The theory then predicts a localization transition which should be contrasted from the glass-transition scenario. Within the theory a divergent localization length emerges and directly 
at the transition anomalous transport is found, reminiscent of the Lorentz model discussed earlier by Götze et al. [41,42]. However, for small concentrations of the fluid component the model should reduce to the Lorentz model, where a gas of noninteracting tracers explores a disordered host structure. There it has been shown that MCT yields inconsistent results, since the singular behavior of the long-wavelength modes is not properly accounted for within the theory [43]. Thus it appears that MCT in its current form is not suited to address all aspects of the localization transition in the Lorentz model or variants thereof [1].

The first goal of this article is to provide an overview on recent progress on slow dynamics of particles confined to disordered host structures. In particular, we will discuss the ramifications of an underlying percolation transition on the dynamics of tracer particles and elucidate various facets of complex transport emerging in the vicinity of the transition. The problem is addressed mostly in terms of computer simulations for different microdynamics. The theoretical analysis is then within a dynamic scaling hypothesis as suggested from dynamical critical phenomena. Also included is a brief discussion of novel data for a tracer in the host matrix that is subjected to an external force. There is also analytic progress for low obstacle densities for a lattice variant of the Lorentz model where the tracer particle is exposed to an external strong driving field.

A second goal is a summary of recent progress on slow dynamics of confined liquids. Here a mode-coupling theory has been formulated [44] that accounts for a slab-geometry. Then the separation of the plates introduces a new length scale into the problem and new physics is expected once this separation becomes comparable to a few particle diameters.

\section{Obstructed dynamics in Lorentz models}

The basic paradigm of hindered motion by frozen obstacles has been formulated by Lorentz [45] where particles explore a disordered array of hard scatterers. While Lorentz conceived the model originally to explain the resistance of metals for electron transport, these Lorentz models have become nowadays valuable minimal models for various transport phenomena in random structures, in particular, for porous media characterized by networks or compartments weakly connected by channels. The importance of Lorentz models stems from various branches of natural sciences where strongly heterogeneous materials are involved. Therefore a physical understanding of the impact on disorder on the macroscopic properties, in particular, particle transport, fluid flow or permeability, is of prime importance for applications in material science, nanochemistry, oil recovery, and more recently for biological systems. Often transport in these disordered structures becomes anomalous in the sense that the mean-square displacement of a tracer particle does not increase linearly for long times, rather it follows a power law $\propto t^{\alpha}$ with an anomalous exponent $\alpha<1$. Examples for such anomalous behavior are ubiquitous in nature and include tracer dynamics in porous soil columns [46], slow transport of alkali ions in nanoporous supercooled silica melts [26,28-30], and subdiffusive motion of proteins in biological matter [47]. In these biological systems the dense packing of differently sized proteins, lipid, sugars, and membranes, is coined macromolecular crowding [48] and leads to a strong suppression of transport with molecular weight [49] of the agents, often eventually giving rise to anomalous diffusion [50] observed in eukaryotes, bacteria, and synthetic biological fluids, see reference [47] for a review.

Here we are concerned with the simplest versions of Lorentz models either in continuum or on a lattice and review the peculiar transport properties emerging via frozen disorder explored by a single tracer particle. 


\subsection{Continuum Lorentz models}

\subsubsection{Structure and void space}

The disordered host structure in continuum is specified by placing obstacles into $d$-dimensional space, such that the tracer can access only regions of space that are not excluded by the obstacles. Correspondingly, the configuration space of the tracer consists of the void space of the obstacle structure. To simplify even more, the obstacles are distributed randomly, identically, and independently, which implies that the host structures are realizations of a spatial Poisson process with number density $n$. Furthermore, the obstacles are considered as spherical such that the tracer cannot come closer to the center of an obstacle than an exclusion distance $\sigma$. Since the obstacles are distributed independently, the exclusion regions may overlap and form clusters. The model is also referred to as Swiss-cheese model where the obstacle clusters correspond to the holes in the cheese while the tracers have to move within the cheese. Then the structural properties of the host matrix are characterized entirely in terms of the dimensionless number density $n^{*}=n \sigma^{d}$. Equivalently one may use the volume fraction accessible to the tracer $\varphi$, often called the porosity or void fraction of the system. From the Poisson process one works out easily that

$$
\varphi=\exp \left(-V_{d} n^{*}\right),
$$

where $V_{d}=\pi^{d / 2} / \Gamma(d / 2+1)$ denotes the volume of the $d$-dimensional unit sphere.

Already at intermediate obstacle densities, the void space is strongly compartmentalized, consisting of pockets of various sizes. Correspondingly, long-range transport is restricted to the component of the void space spanning the entire system. This incipient percolating cluster ceases to exist beyond a certain density $n_{c}^{*}$ known as the percolation threshold. Computer simulations yield $n_{c}^{*} \approx 0.359$ [51] in the twodimensional case, and $n_{c}^{*} \approx 0.838$ [52-55] in 3D. The volume fraction $\varphi_{\infty}$ of the incipient percolating cluster plays the role of an order parameter here and is anticipated to vanish as $\varphi_{\infty} \sim(-\epsilon)^{\beta}$ for $\epsilon \rightarrow 0, \epsilon<0$, where $\epsilon=\left(n-n_{c}\right) / n_{c}$ denotes the separation parameter. More generally, for finite systems of linear dimensions $L$ the volume fraction is expected to obey the finite-size scaling

$$
\varphi_{\infty}(\epsilon, L)=L^{-\beta / \nu} \hat{\varphi}_{\infty}(L / \xi),
$$

where $\xi \sim|\epsilon|^{-\nu}$ denotes the divergent correlation length characterizing the linear dimension at which the infinite cluster becomes homogeneous. Systems much larger than the correlation length, $L \gg \xi$, should be indistinguishable from an infinite system, thus $\varphi_{\infty}$ should become independent of the system size. This implies that the scaling function should behave as a power law $\hat{\varphi}_{\infty}(x \gg 1) \sim x^{\beta / \nu}$ for large dimensionless arguments $x=L / \xi$. Conversely, whenever the correlation length exceeds the box length significantly, the volume fraction is no longer dependent on the correlation length, suggesting that $\hat{\varphi}_{\infty}(x \ll 1)=$ const. approaches a finite value. This entails that directly at the critical point, $n^{*}=n_{c}^{*}$, the volume of the incipient percolating cluster $\varphi_{\infty} L^{d} \propto L^{d_{\mathrm{f}}}$ grows as a power law with the system size, here characterized by the fractal dimension $d_{\mathrm{f}}=d-\beta / \nu$. Correspondingly, the infinite cluster becomes a fractal at the transition, while for obstacle densities below the transition, $n^{*}<n_{c}^{*}$, the infinite cluster is expected to become homogeneous and space-filling at length scales larger than the correlation length $\xi \sim|\epsilon|^{-\nu}$.

In simulations the percolating cluster can be identified by a Voronoi tessellation of the obstacle centers $[53,56]$ which we have implemented relying on the free voro++ library [57]. The measured volume fractions for three-dimensional continuum Lorentz models are displayed in Figure 1 and confirm nicely the theoretical predictions. The 


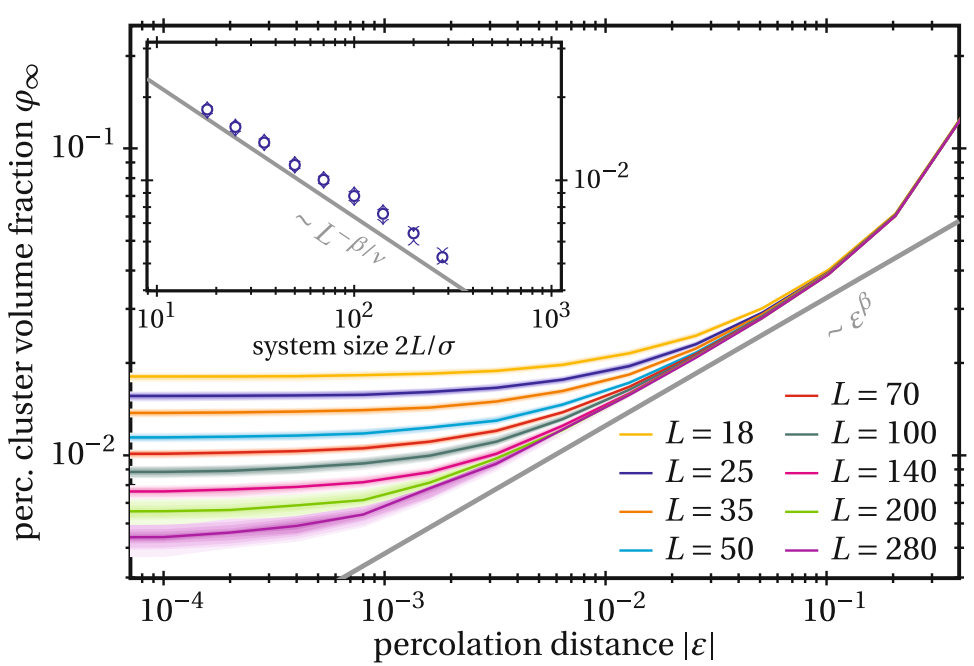

Fig. 1. Volume fraction $\varphi_{\infty}$ of the incipient percolating cluster of the void space between $3 \mathrm{D}$ overlapping spheres as a function of the separation parameter $\epsilon:=\left(n^{*}-n_{c}^{*}\right) / n_{c}^{*}$ from the percolation transition for different system sizes $2 L / \sigma$. Lines represent the median, tinted areas indicate statistical variance among the samples. The power law $\varphi_{\infty} \sim(-\epsilon)^{\beta}$ with $\beta=0.418$ is highlighted by the gray line. In the inset, the value of $\varphi_{\infty}$ directly at the percolation transition $(\epsilon=0)$ is shown as a function of $L$. The expected scaling $\varphi_{\infty} \sim L^{-\beta / \nu}$ is indicated by a gray line.

values of the critical exponent for the growth of the infinite cluster $\beta \approx 0.418$ and the divergence of the correlation length $\nu \approx 0.88$ are consistent with the static exponents for percolation in $3 \mathrm{D}$.

\subsubsection{Mean-square displacement and diffusion}

The dynamics of a tracer particle on this infinite cluster will reflect the fractal properties of the system. The tracer will probe the many dead ends of the fractal before reaching the relevant connections between the compartments to explore finally the entire infinite cluster. This problem of single-particle transport on a fractal structure has been coined by de Gennes [58] as the ant in the labyrinth and one anticipates anomalous transport

$$
\delta r_{\infty}^{2}(t ; \epsilon=0) \sim t^{2 / d_{\mathrm{w}}}, \quad \text { for } t \rightarrow \infty
$$

for the long-time increase of the mean-square displacement $\delta r_{\infty}^{2}:=\left\langle[\boldsymbol{R}(t)-\boldsymbol{R}(0)]^{2}\right\rangle_{\infty}$ of tracers exploring only the percolating cluster. Here the subdiffusive increase is characterized by the exponent $d_{\mathrm{w}}$ which is known as the walk dimension. More generally, one expects that the tracer explores a region of linear dimension $R$ in a typical time $t \sim R^{d_{\mathrm{w}}}$.

For densities below the percolation transition, $\epsilon<0$, the infinite cluster becomes homogeneous on scales larger than $\xi \sim|\epsilon|^{-\nu}$ such that the mean-square displacement should grow as in ordinary diffusion

$$
\delta r_{\infty}^{2}(t, \epsilon<0)=2 d D_{\infty} t, \quad \text { for } t \rightarrow \infty,
$$

where the diffusion coefficient $D_{\infty}=D_{\infty}(\epsilon)$ depends sensitively on the distance to the percolation threshold. For small but nonzero $\epsilon<0$ the mean-square 


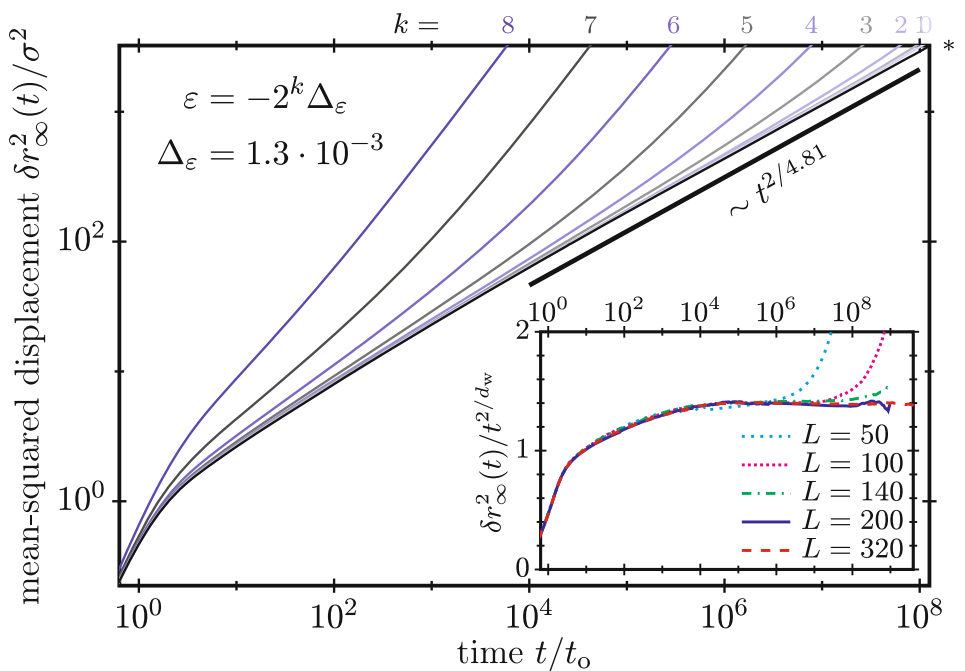

Fig. 2. Double-logarithmic representation of the mean-square displacements $\delta r_{\infty}^{2}(t)$ for ballistic tracer particles on the infinite cluster for the 3D Lorentz model. Distances are measured in units of the exclusion radius $\sigma$, times in units of $t_{\mathrm{o}}=\sigma / v$ where $v$ is the velocity of the tracer. Obstacle density $n^{*}=n_{c}^{*}(1+\epsilon)$ increases from top to bottom in geometric progression to the critical point. The straight line indicates anomalous transport with a walk dimension of $d_{\mathrm{w}}=4.81$. The inset displays a rectification of the data at the percolation threshold for increasing system size.

displacement should follow the anomalous increase $\sim t^{2 / d_{\mathrm{w}}}$ of the fractal cluster up to times $t_{\xi} \sim \xi^{d_{\mathrm{w}}} \sim(-\epsilon)^{-\nu d_{\mathrm{w}}}$ and smoothly cross over to diffusion. This insight suggests that dynamical scaling in the form

$$
\delta r_{\infty}^{2}(t, \epsilon)=t^{2 / d_{\mathrm{w}}} \delta \hat{r}_{\infty}^{2}\left(t / t_{\xi}\right),
$$

should hold. For short rescaled times the scaling function assumes a constant $\delta \hat{r}_{\infty}^{2}(\hat{t} \ll$ $1)=$ const., since the particle has not explored the infinite cluster on times large enough to detect deviations from the fractal behavior. Conversely, for long times diffusive behavior is recovered imposing $\delta r_{\infty}^{2}(\hat{t} \gg 1) \sim \hat{t}^{1-2 / d_{\mathrm{w}}}$, which entails the scaling prediction for the long-time diffusion coefficient $D_{\infty} \sim t_{\xi}^{2 / d_{\mathrm{w}}-1} \sim \xi^{2-d_{\mathrm{w}}}$. In terms of the separation parameter $\epsilon=\left(n_{c}-n_{c}^{*}\right) / n_{c}^{*}$ this is equivalent to $D_{\infty} \sim(-\epsilon)^{\mu_{\infty}}$ with conductivity exponent $\mu_{\infty}=\nu\left(d_{\mathrm{w}}-2\right)[59,60]$.

Simulation data of ballistic tracers in the 3D Lorentz model [61,62] are displayed in Figure 2 and show anomalous transport for the mean-square displacement at the critical obstacle density over more than 6 decades in time. The measured exponent $d_{\mathrm{w}}=4.81$ is in agreement with a theoretical prediction $d_{\mathrm{w}}=d_{\mathrm{f}}+(d-1) / \nu$ by Machta and Moore [63]. The long-time diffusion coefficients $D_{\infty}$ have been shown to vanish as $D_{\infty} \sim(-\epsilon)^{\mu_{\infty}}[62]$ as the transition is approached, corroborating furthermore the exponent relation for the conductivity exponent. Similarly, the scaling hypothesis, equation (5), has been tested by superimposing simulation data $t^{-2 / d_{\mathrm{w}}} \delta r_{\infty}^{2}(t, \epsilon)$ versus rescaled times $t / t_{\xi}$. It appears that convergence to the scaling law is significantly slower $[51,54,62]$ than for the exponents emphasizing that scaling behavior is a more stringent condition on the dynamics than power-law behavior. However, the corrections to scaling are anticipated to be again universal and a considerably better data collapse is achieved by incorporating these corrections in terms of the known exponent for the corrections of the cluster-size distribution $[62,64]$. 
The mean-square displacement $\delta r_{\infty}^{2}(t)$ encodes the same information as the time-dependent diffusion coefficient $D_{\infty}(t):=(1 / 2 d) \mathrm{d} \delta r_{\infty}^{2}(t) / \mathrm{d} t$, the velocityautocorrelation function $Z_{\infty}(t):=\mathrm{d} D_{\infty}(t) / \mathrm{d} t$, or the associated frequency-dependent conductivity $Z_{\infty}(\omega)=\int_{0}^{\infty} \exp (i \omega t) Z(t) \mathrm{d} t$, each quantity being sensitive to a different aspect of the transport phenomenon. The scaling hypothesis for the mean-square displacement, equation (5), translates to each of these cases and has been tested, e.g. to discuss the crossover in the frequency-dependent linear response from dispersive to Ohmic transport $[61,62]$.

Rather than considering only particles on the infinite cluster, one can also investigate the dynamics for tracers starting anywhere in the void space of the host structure $[54,65]$. In essence, the scaling properties also transfer to the corresponding all-cluster average $\delta r^{2}(t, \epsilon)$, the most important modification is now that the anomalous transport at the percolation threshold $\delta r^{2}(t) \sim t^{2 / z}$ becomes slower by admixing the contributions of the finite clusters [66]. The dynamic exponent can be shown to fulfill the scaling relation $z=\nu d_{\mathrm{w}} /(\nu-\beta / 2)$ yielding $z=6.25$ for the $3 \mathrm{D}$ ballistic Lorentz model.

\subsubsection{Non-Gaussian parameter}

While the mean-square displacement provides a first indicator of transport properties in these porous structures, higher moments of the fluctuating displacement $\Delta \boldsymbol{R}(t)=\boldsymbol{R}(t)-\boldsymbol{R}(0)$ encode also valuable information. The next non-trivial quantity is the mean-quartic displacement $\delta r_{\infty}^{4}(t):=\left\langle|\Delta \boldsymbol{R}(t)|^{4}\right\rangle_{\infty}$ where we again only consider tracer particles on the infinite cluster. For a Gaussian process, i.e. if the displacement $\Delta \boldsymbol{R}(t)$ is normally distributed, the mean-quartic displacement can be trivially related to the mean-square displacement. A convenient measure to quantify the deviations from Gaussian transport is the non-Gaussian parameter [47]

$$
\alpha_{2}^{(\infty)}(t)=\frac{d}{d+2} \frac{\delta r_{\infty}^{4}(t)}{\left[\delta r_{\infty}^{2}(t)\right]^{2}}-1,
$$

and a corresponding quantity for the all-cluster averaged quantities. Particles confined to the infinite cluster will eventually display Gaussian transport on length scales larger than the correlation length $\xi$ with corresponding crossover time $t_{\xi} \sim \xi^{d_{\mathrm{w}}}$. Therefore for obstacle densities below the percolation threshold $\alpha_{2}^{(\infty)}(t \rightarrow \infty)=0$, while directly at the transition a non-trivial finite value for $\alpha_{2}^{(\infty)}(t \rightarrow \infty)$ is found [62]. The situation is rather different once all-cluster averages are considered. The reason is that transport becomes a heterogeneous mixture of the different clusters. As a consequence the mean-quartic displacement $\delta r^{4}(t)$ grows faster than the square of the mean-square displacement $\left[\delta r^{2}(t)\right]^{2}$ such that the non-Gaussian parameter $\alpha_{2}(t)$ diverges as a power law directly at the transition with an exponent that can be related to the static and dynamic critical exponents $\nu, \beta, z[54,62]$.

\subsubsection{Space-resolved transport}

The low-order moments of the fluctuating displacement provide valuable temporal information, however, the underlying length scales governing the transport phenomena are only indirectly encoded. To reveal the spatio-temporal dynamics one has to rely on variables that probe the particle trajectories also on a predefined length scale. Typically this is achieved in inelastic scattering experiments [67], e.g. neutron scattering, X-ray, or light scattering, which monitor fluctuations of wavenumber $q$. The measurable quantity is then the intermediate scattering function (ISF) 
$F(q, t):=\langle\exp [i \boldsymbol{q} \cdot \Delta \boldsymbol{R}(t)]\rangle$ where $\boldsymbol{q}$ is the scattering vector connected to the scattering angle which can be adjusted in the experiment. Recently, it has been shown that the ISF can also be obtained from advanced image correlation spectroscopy such as dynamic differential microscopy (DDM) [36,68,69], a method that is also suited to resolve the motion of active particles $[70,71]$. Here we focus again on tracer particles that are located on the infinite cluster only and modify the definition of the ISF accordingly to

$$
F_{\infty}(q, t)=\langle\exp [i \boldsymbol{q} \cdot \Delta \boldsymbol{R}(t)]\rangle_{\infty} .
$$

Close to the critical obstacle density $n_{c}^{*}$ the dynamic scaling hypothesis suggests that the density enters the ISF only in terms of the characteristic length scale $\xi$ and associated time scale $t_{\xi} \sim \xi^{d_{\mathrm{w}}}$

$$
F_{\infty}(q, t, \epsilon)=\hat{F}_{\infty}\left(t / t_{\xi}, q \xi\right)
$$

For small wavenumbers $q \xi \ll 1$ and large times $t \gg t_{\xi}$, the tracer explores a homogeneous medium and ordinary diffusion is anticipated $F_{\infty}(q, t)=\exp \left(-D_{\infty} q^{2} t\right)$. This is compatible with the scaling hypothesis only if $D_{\infty} q^{2} t \sim\left(t / t_{\xi}\right)(q \xi)^{2}$ or $D_{\infty} \sim \xi^{2} t_{\xi}^{-1}$ as we found earlier.

The interesting regime is if the wavenumber probes lengths much larger than the microscopic scale but still small compared to the correlation length, $q \xi \gg 1$. Then the particle explores only regions where the infinite cluster appears fractal, hence it is indistinguishable from the critical point. A similar condition has to be imposed on the time scales, viz. times are long compared to any microscopic scale but small to the characteristic time to sense the finite correlation length, $t \ll t_{\xi}$. In this case the ISF has to be independent of the correlation length and relaxes on the characteristic time scale $\tau_{q}:=t_{\xi}(q \xi)^{-d_{\mathrm{w}}} \sim q^{-d_{\mathrm{w}}}$. Concomitantly the ISF simplifies in the critical regime to

$$
F_{\infty}(q, t)=\mathcal{F}_{\infty}\left(t / \tau_{q}\right) .
$$

The long-time behavior of the ISF can be connected to the probability to return or remain near the starting point [72], similar to the principle idea of fluorescent correlation spectroscopy [73]. Then an algebraic long-time decay $F_{\infty}(q, t) \sim t^{-d_{\mathrm{f}} / d_{\mathrm{w}}}$ is anticipated for small wavenumbers. By the scaling hypothesis, equation (9), this entails that the prefactor increases as $q^{-d_{\mathrm{f}}}$. Hence the ISF reflects both the spatial fractal as well as the temporal fractal. Interestingly, the ratio evaluates to $d_{\mathrm{f}} / d_{\mathrm{w}}=$ 0.525 in $3 \mathrm{D}$ which is close the value $1 / 2$ predicted within a mode-coupling theory [ $41-$ $43,72]$. Simulation results for the ISF for a range of wavenumbers are displayed in Figure 3 and corroborate the theoretical expectation in terms of the dynamic scaling hypothesis. Albeit mode-coupling theory does not capture all facets of the transition, in particular, it misses the fractal nature of the underlying space, the theory is in qualitative and for certain aspects even in semi-quantitative agreement.

\subsubsection{Quenched host structures}

The assumption of independently distributed obstacles is certainly an oversimplification for realistic porous host structures. For example, for the alkali silica melts [26-29] the preferential diffusion pathways for the alkali ions emerge as a quasi-arrested defect structure of a tetrahedral network. Similarly, for size-disparate soft [31] or hard spheres [36] the interaction between the particles leads to strong short-range correlations which modify locally the structure of the pores. Nevertheless fingerprints of the anomalous dynamics as predicted for the Lorentz models are clearly found in all these 


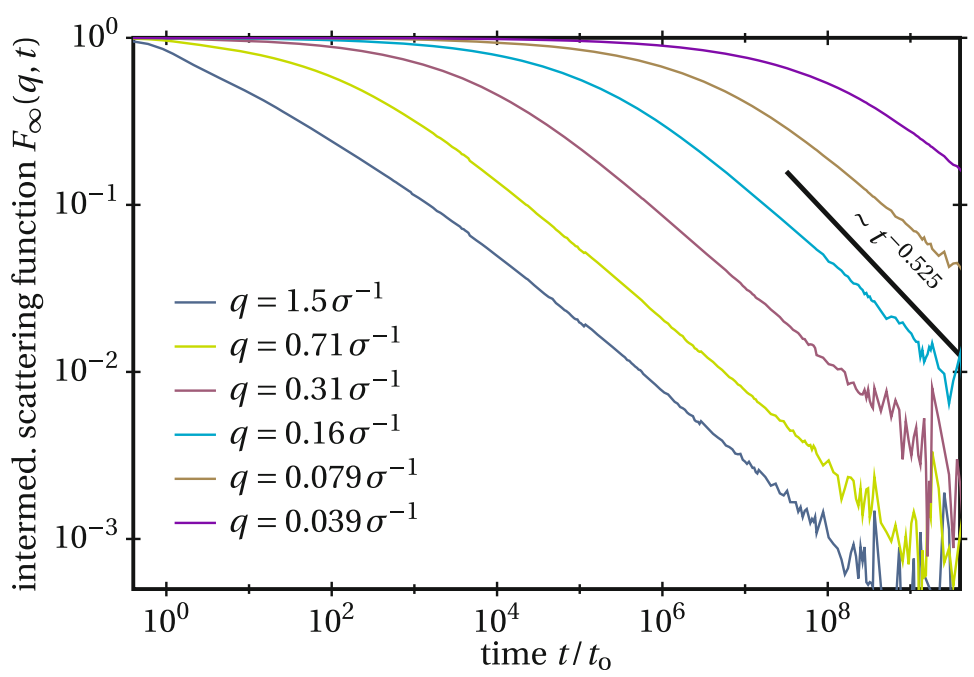

Fig. 3. Intermediate scattering function $F_{\infty}(q, t)$ for the ballistic Lorentz model in 3D for particles on the infinite cluster only. The obstacle density is tuned to the percolation threshold. The terminal relaxation approaches a power law for small wavenumbers $q$, which are measured in terms of the inverse exclusion distance $\sigma^{-1}$. The solid black line represents a power law $\sim t^{-d_{\mathrm{f}} / d_{\mathrm{w}}}$ as guide to the eye.

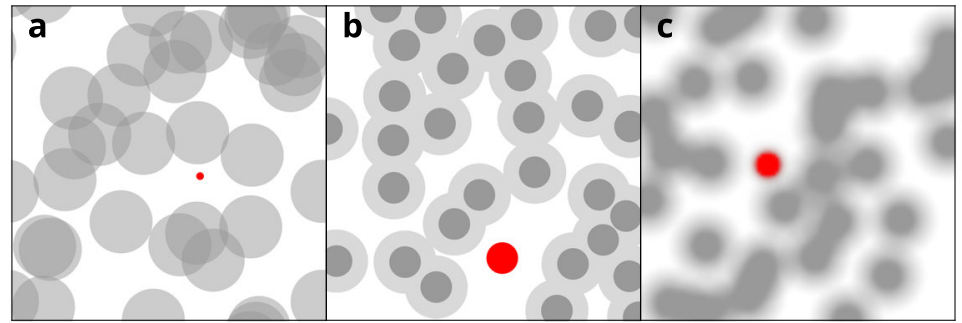

Fig. 4. $(\mathrm{a}-\mathrm{c})$ Illustrations of the considered models. (a) Lorentz model: overlapping obstacles (gray) and point tracer (red). (b) Cherry-pit model: obstacles (dark gray) and extended tracer (red). The area unreachable by the tracer center is marked in light gray. (c) WCAsystem: soft obstacles (gray) and soft tracer (red). Figure reproduced from reference [76] (CC BY 3.0).

systems. Recent experiments $[74,75]$ on quasi-two-dimensional colloidal systems composed of superparamagnetic particles demonstrate that the physics of the localization transition can be realized also in laboratory experiments.

In computer simulations the simplifying assumptions of independently distributed obstacles can be easily relaxed and the scenario of the transition can be compared to the scaling predictions as obtained for the idealized Lorentz model. In a recent case study [76] for 2D the overlapping hard obstacles were replaced first by a quenched hard-disk liquid and second by a quenched polydisperse matrix interacting via a Weeks-Chandler-Andersen potential. Figure 4 illustrates the three different cases as a tracer explores a frozen host structure. The first variation is referred to as the cherry-pit model and consists of a quenched equilibrium configuration of hard disks of diameter $\sigma_{\text {core }}$. The dimensionless control parameter is the packing fraction $\eta=$ $\left(N / L^{2}\right) \pi \sigma_{\text {core }}^{2} / 4$ of the cores at number density $N / L^{2}$. The tracer is modeled itself as a hard disk of diameter $\sigma_{\mathrm{T}}$ such that the interaction distance is $\sigma=\left(\sigma_{\text {core }}+\sigma_{\mathrm{T}}\right) / 2$. 
The second dimensionless control parameter determining the percolation of the void space is then again $n^{*}=\left(N / L^{2}\right) \sigma^{2}$. One may represent the model by associating a halo to each obstacle indicating the areas not accessible to the tracer, see Figure 4. Thus the structure consists of non-overlapping cores, yet the halos of the obstacles may overlap. The percolation transition occurs if the tracer cannot move through the entire structure without touching cores or halos.

The percolation threshold $n_{c}^{*}$ in the cherry-pit model then depends explicitly on the packing fraction $\eta$. For fixed packing fraction the percolation threshold can be accurately determined by a Voronoi tesselation [57] upon gradually increasing the size of tracer, thereby discarding more and more links in the tesselation network until this network no longer spans the system. Interestingly, the maximum value $n_{c}^{*}=0.359$ is assumed for the original Lorentz model with independent obstacles, e.g. $\eta=0$. Upon increasing the packing fraction the critical obstacle density decreases, and as a peculiarity in $2 \mathrm{D}$ systems a minimum emerges at $\eta \approx 0.5$ and increases again for even higher packing fractions [76]. This feature is not present in the corresponding $3 \mathrm{D}$ analog [55]. The percolation transition is present for all packing fractions and universality suggests that the underlying geometrical properties are characterized by the same universal exponents, say $\nu$ for the growth of the correlation length $\xi \sim|\epsilon|^{-\nu}$ and $\beta$ for the weight of the infinite cluster $\varphi_{\infty} \sim(-\epsilon)^{\beta}, \epsilon<0$.

The transport properties of the cherry-pit model have been quantified in the allcluster-averaged mean-square displacement $\delta r^{2}(t)=\left\langle\Delta \boldsymbol{R}(t)^{2}\right\rangle$ for densities approaching the extracted critical obstacle density in geometric progression. Directly at the critical point anomalous subdiffusive transport is expected $\delta r^{2}(t) \sim t^{2 / z}$ with a dynamic exponent $z$. To test the universality the local exponent

$$
\gamma(t)=\frac{\mathrm{d} \log \left[\delta r^{2}(t)\right]}{\mathrm{d} \log (t)}
$$

has been computed from the simulation data, see Figure 5, for a rather high packing fraction $\eta=0.6$. Directly at the percolation threshold this local exponent saturates at a value that is only slightly smaller than the one of the $2 \mathrm{D}$ Lorentz model in Brownian dynamics $z=3.036$ [51] or for hopping transport in the lattice Lorentz model [64]. The residual difference is most likely due to the slow convergence for ballistic tracers, which has been observed also for the original 2D Lorentz model [77]. The origin of this slow convergence for ballistic particles in contrast to Brownian tracers is not understood, attempts to rationalize it in terms of corrections to scaling as in reference [64] don't yield a convincing answer [77].

As a next step towards reality the hard disks have been replaced by soft ones in terms of a Weeks-Chandler-Andersen potential. An equilibrated sample is quenched and acts as host structure for soft noninteracting tracer particles, see Figure 4 . The first observation is that for an ideal gas of tracers in the canonical ensemble the localization transition is replaced by a rapid but smooth crossover [76]. This can be easily rationalized since for soft interactions the barriers introduced by the host structures are finite, therefore the question if a tracer meanders through the entire system or not is strongly energy-dependent. It is instructive to visualize the potential energy landscape as seen by the tracer. For low energies of the tracer the accessible configuration space consists only of isolated low-lying valleys, upon increasing the energy these regions grow and start to merge until at a certain threshold a connected region spanning the entire system emerges. Within this picture it becomes clear that the percolation transition itself becomes energy-dependent. Since in the canonical ensemble every tracer is assigned a different energy, a fraction of the tracers will always be localized while another one always percolates through the system. In fact this insight has been corroborated by switching to a microcanonical ensemble of tracers such that all tracers have the same total energy. Then one finds that the 


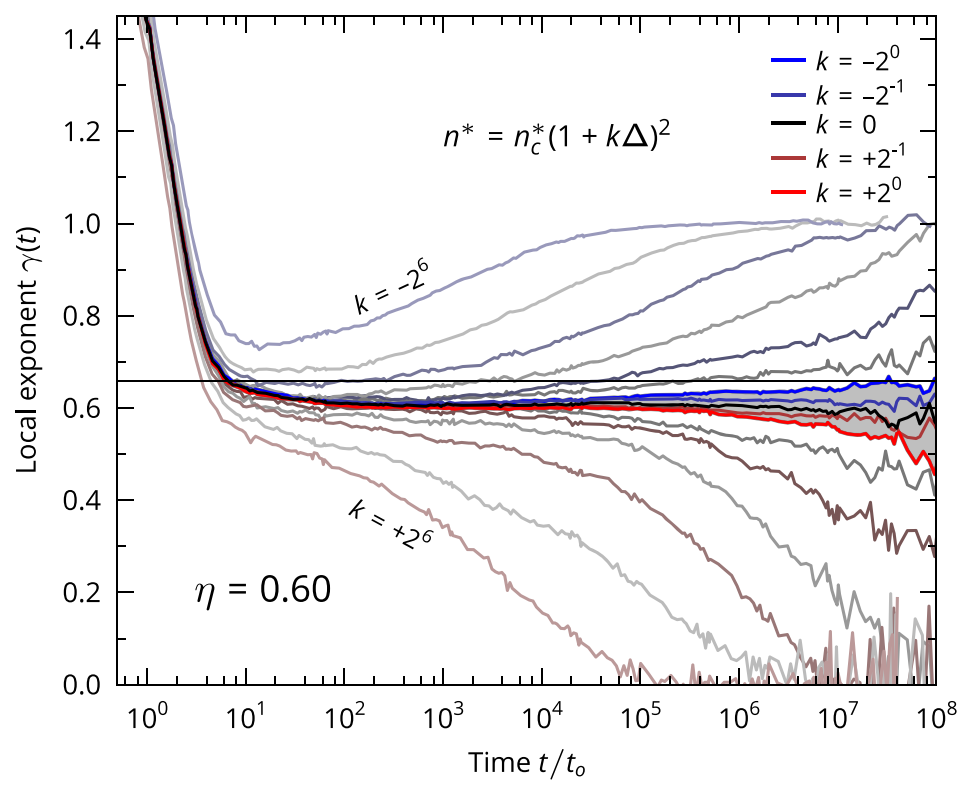

Fig. 5. Local exponents $\gamma(t)=\mathrm{d} \log \left[\delta r^{2}(t)\right] / \mathrm{d} \log t$ of the mean-square displacements $\delta r^{2}(t)$ of the cherry-pit model for the obstacle packing fraction $\eta=0.6$. Reduced density increases from top to bottom. The horizontal line indicates the anomalous exponent $2 / z$ with $z=3.036$ of the Lorentz model. The shaded areas correspond to one standard deviation $\Delta$ in the interaction distance $\sigma$. Figure reproduced from reference [76] (CC BY 3.0).

percolation transition is restored, and anomalous transport becomes pronounced in the vicinity of the threshold and the dynamic exponent is compatible with the one of the 2D Lorentz model [76]. Furthermore the data for the canonical ensemble can be reconstructed as heterogeneous superpositions of the microcanonical ones weighted by the measured distribution of energies.

\subsubsection{Splitting of universality classes}

While the structural properties close to the percolation transition are universal $[53,56]$, irrespective if the system is on a lattice, in continuum with independent or correlated obstacles, this does not necessarily hold for the associated transport processes. Indeed in the theory of continuous phase transitions it is well known that the dynamic universality class describing transport splits into the paradigmatic models A-J $[78,79]$ depending on different conservation laws and couplings. Similarly, for percolative transport the simplest universality class is the one of the random resistor network (RRN) that considers charge transport on a lattice with certain sites blocking the current. Two neighboring unblocked sites are connected by a link and all links have the same conductance.

Universality suggests that it does not really matter if initially the conductances are identical, one may as well start from a distribution of conductances. Upon coarse graining this distribution will renormalize and display a peak at some typical value. Iterating the renormalization step the peak will become narrower and narrower such that gradually the distribution of conductances approaches the one of a single allowed value for the conductance. 
However if the distribution of conductances displays initially a power-law tail of weak conductances a different scenario may arise. It may happen that upon coarse graining the power-law tail becomes more and more dominant at the expense of the typical values, such that after many renormalization steps the distribution is selfsimilar at all scales. Then the transport properties at the percolation threshold are dominated by the tail of weak conductances, thereby giving rise to a new dynamic universality class [80]. More precisely, if the distribution $\rho(\Gamma)$ of conductances $\Gamma$ scales as $\rho(\Gamma) \sim \Gamma^{-\alpha}, 0<\alpha<1$, for $\Gamma \rightarrow 0$, the walk dimension obeys the exponent relation

$$
d_{\mathrm{w}}=\max \left\{d_{\mathrm{w}}^{\mathrm{lat}}, d_{\mathrm{f}}+[\nu(1-\alpha)]^{-1}\right\}
$$

where $d_{\mathrm{w}}^{\text {lat }}$ is the universal exponent for Boolean RRNs and for diffusion on lattices $[59,64,80]$. Thus the weak conductances dominate the transport properties for a sufficiently large exponent $\alpha$.

In the Lorentz model in continuum narrow channels emerge naturally since the obstacles can come arbitrarily close to each other, see Figure 4. Interestingly for the 2D Lorentz model the universality class of the RRN takes over, the narrow channels are irrelevant in the sense of the renormalization group. Therefore the 2D Lorentz model, the $2 \mathrm{D}$ cherry-pit model, and the $2 \mathrm{D}$ soft-sphere model of the previous subsection all display the same critical behavior as the RRN on a lattice. This is markedly different for the 3D Lorentz model where for ballistic tracers the measured value $d_{\mathrm{w}}=4.81$ is larger than the universal lattice value $d_{\mathrm{w}}^{\text {lat }}=3.88$. In fact the measured value $[54,62,81]$ is consistent with a prediction by Machta and Moore [63] suggesting a value of $\alpha=1 / 2$ for the distribution of small transition rates in the 3D Lorentz model.

Since the weak links have been identified as relevant for the $3 \mathrm{D}$ Lorentz model, it is interesting to revisit universality and to investigate the role of the narrow channels. The statistics of having a narrow channel can be modified by introducing structural correlations into the system. Relying on a quenched hard-sphere host structure the probability to find such narrow channels becomes larger than for the uncorrelated Lorentz gas [55]. This observation holds both for the infinite cluster only as well as averaged over all pores of the system. Yet, apart from changing the value of this probability density the changes induced by the packing are smooth deformations with respect to the original Lorentz model. Simulation results for the mean-square displacement $\delta r_{\infty}^{2}(t)$ for ballistic tracers confined to the infinite cluster reveal that the walk dimension $d_{\mathrm{w}}$ remains the same for all packing fractions [55]. Therefore the conclusion is that the statistics of the narrow channel is irrelevant as long as the probability density extrapolates to a constant for small channel widths. However, changing the dynamics from ballistic to Brownian tracers does have an impact on the walk dimension, here the mean-square displacement $\delta r_{\infty}^{2}(t) \sim t^{2 / d_{\mathrm{w}}}$ increases with a smaller value of the walk dimension of $d_{\mathrm{w}}=4.24$. Thus the way the narrow channels are probed is important and surprisingly Brownian tracers are faster than ballistic ones.

For both cases one can estimate the typical transition rates between two compartments connected by a narrow channel. Assuming that for ballistic tracers the channel acts as a pinhole, i.e. everything that hits goes through, the typical transition rate should be proportional to the cross-sectional area $\sim w^{2}$ where $w$ is the width of the channel. Similarly, for Brownian particles the channel should act as an Ohmic resistor, such that the typical transition rate is proportional to the cross-sectional area and inversely proportional to the effective length $L_{w}$ of the channel. Here $L_{w}$ estimates on what scale the narrow channel remains narrow, and by Pythagoras one works out that $L_{w} \sim \sqrt{w}$. Collecting results the prediction for Brownian tracers is that the typical transition rate should scale as $\sim w^{3 / 2}$. Using these expressions to convert the 


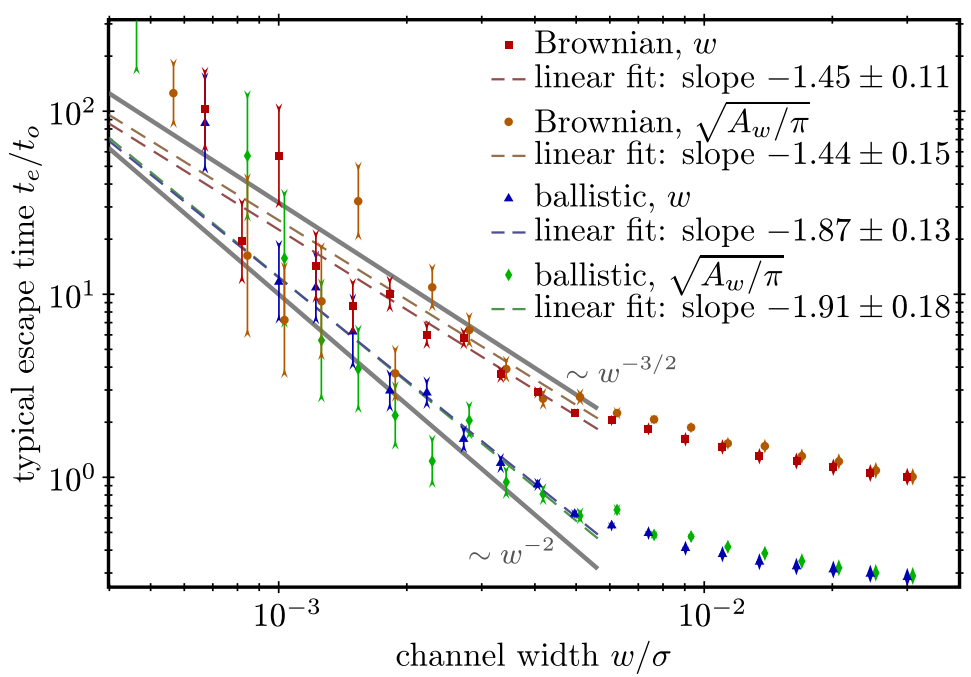

Fig. 6. Typical escape time $t_{e}$ of a tracer particle exiting from a Delaunay simplex through a narrow channel of effective width $w \ll \sigma$ using either ballistic or Brownian dynamics. Included are the typical escape time in terms of an effective circular channel width $\sqrt{A_{w} / \pi}$ (red and orange data points) obtained from the measured cross-sectional areas $A_{w}$. A clear splitting between the scaling of the typical escape time for ballistic and Brownian motion emerges and the fitted exponents differ by 0.5. For comparison, the expected power laws with exponents -2 (ballistic) and $-3 / 2$ (Brownian) are indicated by solid gray lines. Figure reproduced from reference [55] (C) 2016 American Physical Society. This figure is subject to copyright protection and is not covered by a Creative Commons License.)

distribution of narrow channels to probabilities for small transition rates one finds the values $\alpha=1 / 2$ [63] for ballistic tracers and $\alpha=1 / 3$ [82] for Brownian ones, which explains the measured values for the walk dimension.

The distribution of escape times from a compartment can also be extracted directly from the simulation and can be fitted reasonably well by a log-normal distribution. In particular, the mean value may differ from the typical value by orders of magnitude. From the fit the position of the maximum is used as a typical escape time, see Figure 6 . Then one observes that indeed the typical escape times grow as predicted theoretically in the limit of small channel widths, thus demonstrating the splitting of the dynamic universality class of percolative transport.

It is interesting to investigate whether further universality classes beyond the ballistic and the Brownian dynamics exist. A natural candidate are ballistic tracers for soft obstacles, such that the narrow channels are replaced by passes in the energy landscape that lie barely below the total energy. A second example may be Brownian motion in suspension such that hydrodynamics introduces lubrication forces rendering the small channels more difficult to pass. A third somewhat more remote example is 2D transport of particles undergoing circular orbits which are reflected elastically at the obstacles. There it has been shown that a delocalization transition at low density emerges $[83,84]$ in addition to the conventional localization transition where small transition rates between barely connected clusters naturally occur.

\subsubsection{Driven systems}

The dynamical properties of the tracer in equilibrium provide valuable information also on the linear susceptibilities by the fluctuation-dissipation theorem. For example, if an external force $F$ acting on the tracers is switched on at a certain instant of time, 
say at time $t=0$, the tracers will respond by a net movement along the direction of the force. For small forces one anticipates that this time-dependent drift velocity $v(t)$ is linearly related to the force and the detailed connection is provided by the fluctuation-dissipation theorem

$$
v(t)=\frac{F}{k_{B} T} \int_{0}^{t} Z\left(t^{\prime}\right) \mathrm{d} t^{\prime}+o(F)
$$

Here $Z(t)$ denotes the velocity-autocorrelation function in equilibrium and $k_{B} T$ is the thermal scale. Equation (12) generalizes the Einstein relation for the steady state drift velocity $v(t \rightarrow \infty)=D F / k_{B} T$ via the Green-Kubo relation $D=\int_{0}^{\infty} Z(t) \mathrm{d} t$. Generalizations for the linear response to arbitrary time dependences of the force are straight forward and yield frequency-dependent mobilities as linear susceptibilities.

Here we want to discuss briefly what happens beyond the linear response in the Lorentz model, i.e. if the forces are not small anymore. Since the diffusion coefficients become arbitrarily small as the percolation transition is approached, the linear response becomes smaller and smaller. At the same time one anticipates that the linear response regime shrinks to smaller and smaller forces. Directly at the transition the linear response strictly vanishes since transport remains anomalous for all times implying that the diffusion coefficient is zero. Nevertheless the drift velocity will not vanish and by self-similarity a power-law dependence is anticipated.

Achieving accurate simulation results for the driven Lorentz model turns out to be extremely difficult. First the nonequilibrium steady state is not known but it has to be reached within the simulation time. However as the critical point is approached the relaxation time $t_{\xi} \sim \xi^{d_{\mathrm{w}}}$ diverges such that it takes longer and longer to reach the steady state. Second, since the linear-response regime shrinks the applied forces have to be tiny to see the crossover to the linear response. Correspondingly long simulation runs have to be performed to still extract a net drift velocity beyond the noise. There is an additional conceptional problem of the driven Lorentz model for the case of ballistic tracers. Here the particles gain energy by descending the potential and thus would become faster and faster. This appears to be an artifact of the model since no inelastic collisions can thermalize the system. Therefore one should switch to Brownian dynamics where the velocity fluctuates always around the thermal velocity no matter how far the motion along the field actually went.

Physically one expects that by the forces the tracers are drawn in dead ends of the ramified structures and the tracer will reside in these pockets for exponentially large times until a thermal fluctuation helps the particle to climb all the way up against the gradient to find the right pathway through the structure. Clearly only particles located on the infinite cluster can contribute to a non-vanishing drift velocity.

Results for the stationary drift velocity as a function of the external force are displayed in Figure 7. For obstacle densities not too close to the percolation transition the linear response regime is reached. Upon increasing the force the data become less and less reliable since convergence to the steady state has not been achieved in all runs. This problem becomes more serious the closer the density is to $n_{c}^{*}$. Empirically we find that a power-law decrease $F^{-\alpha}$ emerges for large forces with an exponent that appears to depend also on the density. A suitable interpolation formula is provided by $v=1 /\left(a / F+b F^{\alpha}\right)$. Currently it remains on open question whether a nonlinear response directly at the transition can be identified and if dynamic scaling holds also in the nonlinear regime. Due to the problems mentioned it may be worthwhile to readdress the problem again on a lattice with hopping dynamics. 


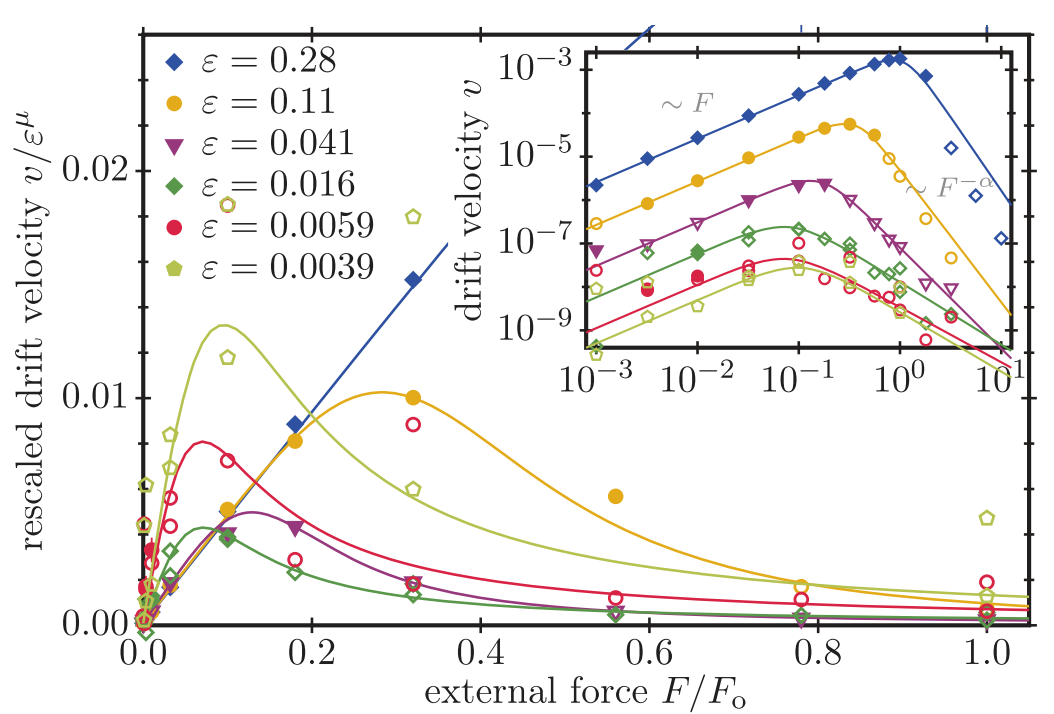

Fig. 7. Stationary drift velocity $v$ of a tracer particle in response to a constant uniform force $F$ for various separation parameters $\epsilon:=1-n^{*} / n_{c}^{*}$. The drift velocities have been rescaled by dividing by the scaling prediction $\epsilon^{\mu}$ in linear response with conductivity exponent $\mu=2.38$ for Brownian particles in 3D. Full symbols indicate that the tracer dynamics has successfully relaxed into its long-time limit, while open symbols point out that no steady drift was reached. Error bars are included for the full symbols. Lines represent an empirical fit of $1 /\left(a / F+b F^{\alpha}\right)$. The inset shows the same data in double-logarithmic representation, highlighting the behavior for small forces $F$.

\subsection{Lorentz gas and the low-density expansion}

Fundamental insight into generic transport properties have been gained in terms of exact solutions of the Lorentz model for low obstacles densities. There each trajectory is decomposed into a sequence of free motions interrupted by scattering events with individual obstacles. Reorganizing the cascade of scattering events in terms of a multiple-scattering expansion reveals that to first order in the obstacle density only the scattering problem with a single obstacle needs to be solved. The first approaches in this direction have been made in the late 1960's in the seminal work of Weijland and van Leeuwen [85]. Since the scattering with a single hard sphere for a ballistic particle is trivial and yields a trajectory that never comes back, an involved resummation procedure had to be introduced for the expansion. In essence, one has to solve first for Boltzmann's equation to account for sequences of independent scattering events, the first-order-density correction then emerges via repeated interactions with the same obstacle where free ballistic motion is replaced by the Boltzmann evolution operator. The solution can be obtained only for large times, long wavelengths, and low densities. A puzzling result is that the diffusion coefficient becomes a nonanalytic function of the obstacle density $[85,86]$. The origin of this phenomenon can be traced back to the divergence of the mean-free path length as the obstacle density becomes small.

Even more bewildering are the ramifications of the repeated scattering for the time- and length-dependent transport properties. The frequency-dependent diffusion coefficient is related by the generalized Green-Kubo relation

$$
D(\omega)=\int_{0}^{\infty} e^{i \omega t} Z(t) \mathrm{d} t
$$


to the velocity-autocorrelation function $Z(t)=(1 / d)\langle\boldsymbol{v}(t) \cdot \boldsymbol{v}(0)\rangle$. In particular, for the stationary state, $\omega=0$, the standard Green-Kubo relation is recovered. Assuming that $Z(t)$ decays exponentially fast yields a frequency-dependent diffusion coefficient that is an analytic function for small frequencies. However it was shown that in the ballistic Lorentz model the velocity-autocorrelation function should behave asymptotically as $Z(t) \simeq-A t^{-(d+2) / 2}$ with a coefficient $A>0[86,87]$. Thus the decay is only algebraic implying persistent anticorrelations by the repeated scattering events with the same obstacle. Loosely speaking, these correlations build up since the particle remembers that the path is blocked by an obstacle, the power-law decay connects to the algebraic decay of the return probability in the diffusion propagator [86]. Simulation results are difficult to obtain in the regime of low densities but confirm both the algebraic decay as well as the prediction for the prefactor [81].

While for the ballistic Lorentz model the velocity-autocorrelation function has been worked out only asymptotically, the full time dependence is accessible for the Brownian case [88]. Even more, explicit expressions for the intermediate scattering function have been provided. In particular the long-time tail of the velocityautocorrelation function is recovered. The key insight is that the involved resummation by solving first for Boltzmann's equation can be avoided since in the Brownian case the tracer returns to the same obstacle already merely by the free diffusion.

\subsubsection{Equilibrium dynamics in the lattice Lorentz model}

Further analytic progress is made by considering obstructed motion of a tracer particle on a lattice. The lattice analog of the Lorentz model considers hopping transport on a disordered lattice such that a certain fraction of sites is occupied by obstacles. Tracers can explore the lattice and hop to neighboring sites with equal rates provided the site is not blocked. The model again displays a percolation transition of the empty lattice sites which coincides with the localization transition of the transport problem. The universality class describing the critical properties is provided by the random resistor network which has been studied by extensive computer simulations in the literature $[59,64]$.

The velocity-autocorrelation function has been solved exactly for all times to first order in the obstacle density by reducing the problem to the interaction with a single scatterer [89]. The main prediction is that again an algebraic decay emerges due to repeated interactions with the same obstacle. These predictions have been confirmed by elaborated computer simulations [90]. Later it became evident that these tails in the velocity-autocorrelation functions are generic [91,92] for an entire class of lattice Lorentz models since the mechanism relies only on the particle conservation law $[86]$.

\subsubsection{Field induced response}

Since the velocity-autocorrelation function of the lattice Lorentz gas can be evaluated explicitly in first order of the obstacle density and for all times, the lattice Lorentz gas constitutes a promising model to investigate the influence of a force on the tracer. In the driven lattice Lorentz gas the system is initially in equilibrium and a uniform step force is switched on at time zero pulling the tracer along a lattice direction $[93,94]$. The dimensionless force $F=$ force $\cdot a / k_{\mathrm{B}} T$ measures the strength of the applied force on the tracer and it introduces a bias in the nearest-neighbor transition probabilities $W(\boldsymbol{d} \in \mathcal{N})$. The transition probabilities fulfill local detailed balance in both lattice directions with $W\left(a \boldsymbol{e}_{x}\right) / W\left(-a \boldsymbol{e}_{x}\right)=e^{F}$ and $W\left(a \boldsymbol{e}_{y}\right) / W\left(-a \boldsymbol{e}_{y}\right)=1$ 

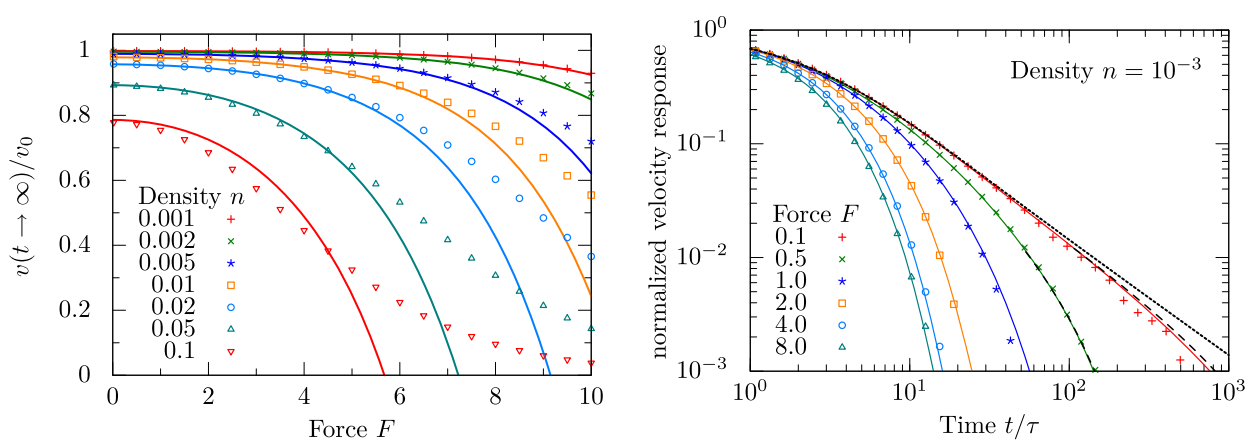

Fig. 8. (Left panel) Stationary velocity in units of the bare velocity $v_{0}=(a / 2 \tau) \sinh (F / 2)$ for different densities and forces. Solid lines correspond to the analytic solution of the stationary velocity in first order of the obstacle density and symbols represent simulation results. (Right panel) Velocity response $v(t)-v(t \rightarrow \infty)$ normalized by it is initial value $t \rightarrow 0$. Solid lines represent the first-order theory and symbols correspond to simulation results with an obstacle density of $n=10^{-3}$. The black dotted line represents the time-dependent behavior predicted by the fluctuations-dissipation theorem. The black dashed lines correspond to the long-time behavior including the exponential decay as predicted by the theory.

and a convenient choice is $W\left( \pm a \boldsymbol{e}_{x}\right)=e^{ \pm F / 2} /\left(e^{F / 2}+e^{-F / 2}+2\right)$ and $W\left( \pm a \boldsymbol{e}_{y}\right)=$ $1 /\left(e^{F / 2}+e^{-F / 2}+2\right)$. The choice of the transition probabilities is not unique and a different dependence has also been considered in the literature $[95,96]$. Unnormalized transition rates such that the exponentially distributed waiting time of the tracer after a jump is characterized by a mean-waiting time of $\tau / \Gamma$ with $\Gamma=[\cosh (F / 2)+1] / 2$ appear also naturally.

Similarly to the lattice Lorentz gas in equilibrium [97-99], the first-order density response can be evaluated by considering all possible interactions of the tracer with a single obstacle. Although the calculations are the same in principle, the formulas become much more involved due to the inclusion of the force and one has to rely on computer algebra to obtain closed expressions [93,94].

The response of the tracer is encoded in the displacement $\Delta x(t)=x(t)-x(0)$ along the force for times where the force acts on the tracer. The first quantity of interest is given by the velocity of the tracer defined by a time derivative of the mean-displacement:

$$
v(t)=\frac{\mathrm{d}}{\mathrm{d} t}\langle\Delta x(t)\rangle,
$$

where the symbol $\langle\cdot\rangle$ denotes an average over all possible obstacle realizations and starting positions of the tracer.

In the absence of obstacles, the tracer velocity is time-independent and readily obtained by the difference of the forward and backward transition rate in a single jump event leading to $v_{0}=(a / 2 \tau) \sinh (F / 2)$. In the presence of obstacles, the velocity becomes time-dependent and the immediate response is determined by the first jump event with $v(t \rightarrow 0)=v_{0}(1-n)$. The stationary velocity of the tracer for long times, can be evaluated explicitly for all forces in first order of the density and is expressed in terms of complete elliptic integrals [93]. For fixed obstacle density and increasing force, the stationary velocity $v(t \rightarrow \infty)$ of the tracer becomes more and more suppressed [Fig. 8 (left panel)]. The same behavior is also found for fixed force and increasing density of obstacles such that the stationary velocity is purely disordersuppressed where an increase in the disorder leads to a decrease of the stationary state velocity. For small forces, the stationary velocity is no longer determined by an 

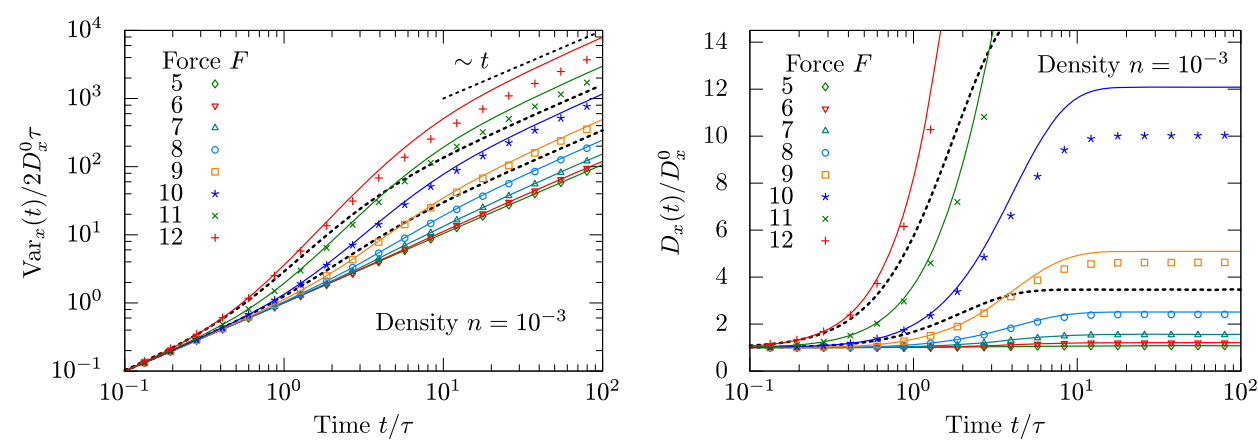

Fig. 9. Time-dependent variance of the tracer displacement parallel to the force for different strength of the driving in the presence of obstacles with a density of $n=10^{-3}$ (left panel). Time-dependent diffusion coefficient parallel to the force (right panel). Force increases from bottom to top. Lines represent the theory and symbols correspond to simulation results. The black dashed lines represent simulation results of a tracer in the presence of mobile obstacles with mean waiting time $\tau$ and forces $F=10$ and $F=12$. Figure reproduced from reference [94] (C) 2017 American Physical Society. This figure is subject to copyright protection and is not covered by a Creative Commons license.)

analytic expansion in the force as in the case of the bare velocity. Instead, the interactions of the tracer with the obstacle disorder introduce logarithmic contributions beyond the linear response behavior.

In the linear response, the time-dependent approach to the stationary state velocity is determined by the velocity-autocorrelation function $Z(t)=(1 / 2) \mathrm{d}^{2}\left\langle\Delta x(t)^{2}\right\rangle / \mathrm{d} t^{2}$ of the tracer in equilibrium:

$$
v(t)=\frac{F}{a} \int_{0}^{t} \mathrm{~d} t^{\prime} Z\left(t^{\prime}\right) .
$$

Since the velocity-autocorrelation function exhibits a slow algebraic decay of the form $Z(t) \sim t^{-2}$ for long times due to the persistent memory introduced by the obstacles, the fluctuation-dissipation theorem predicts an algebraic approach to the stationary velocity $v(t \rightarrow \infty)$ with $\sim t^{-1}$. However, the analytic solution reveals that for any small but finite driving the algebraic decay is decorated by an exponential one such that the stationary-state velocity is always approached exponentially fast [Fig. 8 (right panel)]. The time scale for the exponential decay is set by the applied force and scales as $\sim 1 / F^{2}$.

The fluctuations of the tracer along the force are measured in terms of the variance of the displacements:

$$
\operatorname{Var}_{x}(t)=\left\langle[\Delta x(t)-\langle\Delta x(t)\rangle]^{2}\right\rangle=\left\langle\Delta x(t)^{2}\right\rangle-\langle\Delta x(t)\rangle^{2} .
$$

The corresponding diffusion coefficient $D_{x}(t)$ is then obtained by a time derivative via $D_{x}=(1 / 2) \mathrm{dVar}_{x}(t) / \mathrm{d} t$. In the absence of obstacles, the fluctuations along the force increase diffusively with a bare diffusion coefficient of $D_{x}^{0}=\left(a^{2} / 4 \tau\right) \cosh (F / 2)$. In the presence of obstacles and for increasing strength of the driving, the fluctuations exhibit a pronounced intermediate superdiffusive regime governing the transition to the stationary state [Fig. 9 (left panel)]. In the stationary state, the fluctuations increase diffusively, yet the stationary diffusion coefficient along the force is considerably enhanced [Fig. 9 (right panel)].

The superdiffusively growing fluctuations at intermediate times can be rationalized in terms of an asymptotic model in the limit of large forces [94]. In this case, the transition rates along the field dominate the motion of the tracer and transitions 
perpendicular and against the field can be ignored. Then, the fluctuations of the tracer along the field at intermediate times can be evaluated explicitly to

$$
\operatorname{Var}_{x}(t)=\frac{1}{3} \frac{n a^{2}}{64} \exp (3 F / 2) \frac{t^{3}}{\tau^{3}}, \quad \tau^{*} \lesssim t \lesssim \tau .
$$

The asymptotic model is valid up to times where the diffusion of the tracer perpendicular to the field becomes relevant. The time scale for the onset of the superdiffusion is identified as $\tau^{*} \sim \tau e^{-F / 2} / \sqrt{n}$ and the regime of superdiffusion grows with increasing force. From the asymptotic model, a true superdiffusive exponent of 3 is identified in the limit of large forces which constitutes an upper bound for the increase of the fluctuations at intermediate times.

The transport behavior in the stationary state can also be characterized in terms of the force-induced diffusion coefficient $D_{x}^{\text {ind }}=D_{x}(t \rightarrow \infty)-D_{x}^{\text {eq }}(t \rightarrow \infty)$, with longtime diffusion coefficient $D_{x}^{\mathrm{eq}}(t \rightarrow \infty)=\left(a^{2} / 4 \tau\right)[1-(\pi-1) n]$ in equilibrium [97]. Similarly to the stationary velocity for small forces, the force-induced diffusion coefficient $D_{x}^{\text {ind }}$ acquires nonanalytic contributions which arise solely due to the interactions of the tracer with the obstacle disorder. For large forces, the force-induced diffusion coefficient increases exponentially with $D_{x}^{\text {ind }} \sim \exp (3 F / 2)$ which is also purely obstacle-induced.

Interestingly, the stationary diffusion coefficient $D_{x}(t \rightarrow \infty)$ exhibits two different force regimes in first order of the density where the fluctuations can be either disordersuppressed or disorder-enhanced. In the disorder-suppressed regime, an increase in the disorder leads to a suppression of the fluctuations. For forces $F \gtrsim 1.45$ the fluctuations in the stationary state are disorder-enhanced where an increase in the disorder leads to an enhancement of the growth of the fluctuations. This phenomenon has also been found in other models for driven systems [100-102].

It is interesting to ask if the results obtained for the driven lattice Lorentz model can be transferred to the continuum case. Here the hopping motion is replaced by Brownian motion and the hard obstacles are replaced again by hard spheres in 3D. The master equation for the conditional probability then should be replaced by a suitable many-body Smoluchowski equation. For the case of dilute colloidal suspension, series expansions for the stationary velocity have been elaborated [103], however the full dynamical response remains to be worked out.

\section{Confined liquids}

Understanding the transport properties of confined liquids is of great interest for numerous practical applications. Examples range from liquid supply, adhesion to lubrication, friction in narrow channels as well as in biological systems. Confinement effects for densely-packed liquids have been investigated in slit geometry mainly by computer simulations $[104,105]$ and experiments $[106,107]$ focusing on the regime of moderate confinement with slit widths of several particle diameters or larger. These studies revealed the existence of an oscillatory density profile close to the wall [106]. Furthermore, the dynamics in confinement has been shown to increase or decrease compared to the bulk depending on the roughness of the walls [108,109]. A remarkable empirical scaling of the diffusivities with the excess entropy $[110,111]$ has also been established for confined liquids.

Another recent focus has been given in the study of glasses to introduce competing mechanisms, which, in principle, can lead to a nonmonotonic state diagram. Such kind of reentrant scenarios have been realized upon adding short-range interactions to the colloidal particles $[17,18]$ or inserting the liquid into a frozen disordered 


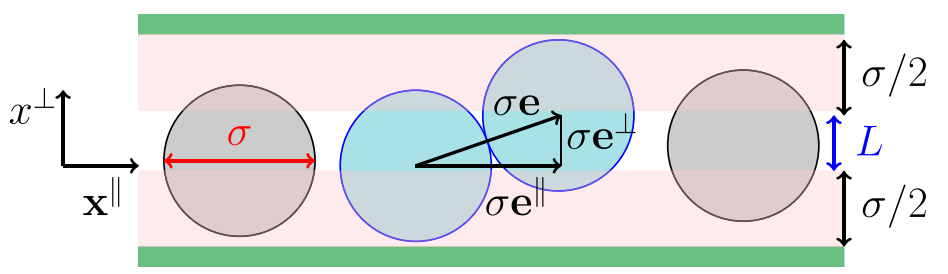

Fig. 10. A schematic cross section of hard spheres of diameter $\sigma$ confined to a slit of accessible width $L$. For two colliding spheres the momentum transfer is almost parallel to the walls in the limit of $L \rightarrow 0$. Figure reproduced from reference [118] (C) 2017 American Physical Society. This figure is subject to copyright protection and is not covered by a Creative Commons license.)

host structure [112,113]. Alternatively, confinement can also introduce such competing mechanisms. To describe dense liquids in such confinement the MCT has been extended $[44,114]$ relying on symmetry-adapted modes that account for the broken translational symmetry perpendicular to the walls. A striking prediction of the MCT in slit geometry has been the emergence of a multiple reentrant glass transition in the nonequilibrium state diagram as a function of the slit width along lines of constant packing fraction $[44,115,116]$. This multiple reentrance is attributed to a complex competition between the layering induced by the walls and the local caging. This scenario has been corroborated by event-driven molecular dynamics simulations for slightly polydisperse hard-sphere systems $[115,117]$ upon measuring the self-diffusion coefficients parallel to the walls.

Here we briefly summarize the recently developed mode-coupling theory in confinement and its nonequilibrium state diagram in comparison to computer simulations. Furthermore, we review the emergence of a divergent time scale controlling the dimensional crossover from $2 \mathrm{D}$ systems to $3 \mathrm{D}$ confined systems.

\subsection{Glassy dynamics in confinement}

\subsubsection{Mode-coupling theory for confined liquids}

The theory considers a single-component liquid comprised of $N$ identical structureless particles of mass $m$ confined between two plane parallel smooth hard walls, see Figure 10. Particle positions are specified by $\boldsymbol{x}_{n}=\left(\boldsymbol{x}_{n}^{\|}, x_{n}^{\perp}\right)$ for $n=1, \ldots, N$, where $\boldsymbol{x}_{n}^{\|}$describes the in-plane coordinates. The confinement restricts the transverse positions to $-L / 2 \leq x_{n}^{\perp} \leq L / 2$. For hard particles with exclusion radius $\sigma / 2$, the physical wall separation then corresponds to $H=L+\sigma$. Since the confinement induces a modulation of the equilibrium density profile $n\left(x^{\perp}\right)$, the fluctuations in the direction perpendicular to the walls are decomposed into a set of discrete Fourier modes characterized by a wavenumber $Q_{\mu}=2 \pi \mu / L, \mu \in \mathbb{Z}$ where $L$ denotes the width of the slit accessible to the particles. The key quantity is the generalized intermediate scattering function $S_{\mu \nu}(q, t)=N^{-1}\left\langle\rho_{\mu}(\boldsymbol{q}, t)^{*} \rho_{\nu}(\boldsymbol{q}, 0)\right\rangle$, where

$$
\rho_{\mu}(\boldsymbol{q}, t)=\sum_{n=1}^{N} \exp \left[\mathrm{i} Q_{\mu} x_{n}^{\perp}(t)\right] \mathrm{e}^{\mathrm{i} \boldsymbol{q} \cdot \boldsymbol{x}_{n}^{\|}(t)}
$$

are symmetry-adapted Fourier modes for the microscopic density, and $\boldsymbol{q}=\left(q_{x}, q_{y}\right)$ are the conventional wave vectors in the lateral plane.

Relying on this set of distinguished variables the equations of motion have been derived within the Zwanzig-Mori projection operator formalism $[44,114,119]$. As a 

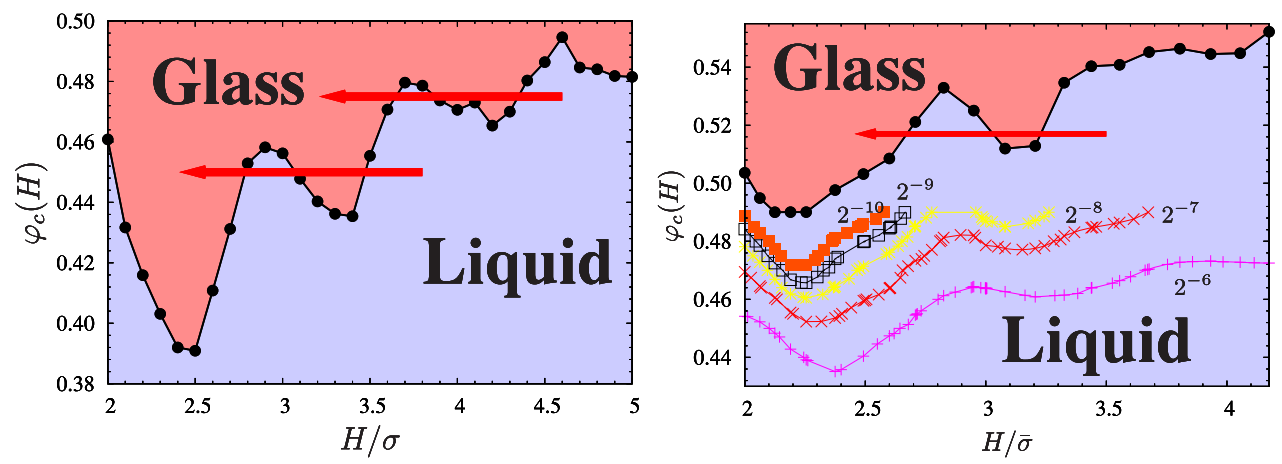

Fig. 11. Nonequilibrium state diagram as obtained from a numerical solution of the MCT equations for a hard-sphere fluid (left panel) and power-law fits of the simulated diffusion coefficients to $D \sim\left(\varphi-\varphi_{c}(H)\right)^{-\gamma}$ (right panel). The right figure also includes the isodiffusivity lines with indicated values. The multiple reentrant is indicated by arrows of constant density. Figures reproduced from reference [115] (C) Nature Communications 2014. This figure is subject to copyright protection and is not covered by a Creative Commons license.)

peculiarity of the confinement there appear now two different relaxation channels corresponding to currents parallel and perpendicular to the walls, thereby changing the mathematical structure of the theory with respect to bulk systems. The equations are closed by a mode-coupling approximation for the memory kernels by projecting onto pairs of generalized density modes. For the tagged-particle motion a similar approach yields equations of motion which couple to the collective density [120].

A natural question that arises is if these equations still provide unique solutions that represent correlation functions as has been proven in bulk systems $[1,121-123]$ and if the long-time limits can be shown to exist [116]. The first question has been answered positively recently for general multiple relaxation channels [124] which arise for example also for molecular systems $[125,126]$.

A second interesting issue is to ask how a narrow slit approaches a $2 \mathrm{D}$ system as the wall separation decreases. There it has been shown that MCT converges under certain conditions to the 2D counterpart [119], however the limits of time to infinity and plate separation to zero do not commute. Therefore one anticipates two different glassy states, one for the 2D system and another one for the confined 3D set-up.

Here we do not repeat the equations of motion but refer to a recent review [117], rather we focus on the nonequilibrium state diagram.

\subsubsection{Comparison to computer simulations}

The nonequilibrium state diagram within MCT has been obtained $[44,115]$ by scanning the parameter space $(\varphi, H=L+\sigma)$ taking the structural input obtained from the inhomogeneous Percus-Yevick closure [67,127-129], see Figure 11. The result demonstrates a striking multiple reentrant behavior. Here, the glassy behavior is suppressed for wall distance of integer values of the particle diameter $\sigma$, while for half-integers the dynamics is already blocked due to incommensurable packing. Consequently, in the latter case only a moderate density is required to force the liquid into a glassy state.

This state diagram is also compared to computer simulations for $10 \%$ polydisperse hard-sphere systems of mean diameter $\bar{\sigma}$. This allows testing the MCT prediction on a semi-quantitative level. The critical packing fraction within the simulations have been determined by power-law fits to the lateral diffusion coefficient $D \sim\left(\varphi-\varphi_{c}(H)\right)^{-\gamma}$, 
with MCT-exponent $\gamma$. Interestingly, the state diagram for polydisperse hard spheres corroborates the multiple reentrant scenario qualitatively. For instance, the oscillations within the transition are weaker and the extrema are slightly shifted to lower wall separations. Another extension of the above reentrant behavior to a wedge geometry with small opening angle predicts a possible liquid-glass coexistence [115].

\subsection{Decoupling in strong confinement}

The regime of extremely confined fluids where only a monolayer fits between the walls has been investigated by computer simulations, density functional theory, integral equations and experiments both for colloidal as well as for molecular liquids $[130,131]$. However, analytical progress in this direction has been achieved only very recently $[119,132-134]$. Within this theoretical study, the key observation was that in strong confinement the canonical ensemble for the fluid in a slit geometry decouples into a two-dimensional fluid in the lateral plane and an ideal gas in the transversal direction. Interestingly, in the limit of extreme confinement a hidden small parameter $n_{0} L^{2}$ has been identified, where, $n_{0}$ denotes the $2 \mathrm{D}$ number density, which allows calculating structural properties systematically.

\subsubsection{Density profile}

It is interesting to elaborate the influence of the transversal degrees of freedom on structural quantities, such as the density profile perpendicular to the walls. In the limit of $L \rightarrow 0$ one can determine the density profile by taking the transversal degrees of freedom as a small perturbation. The density profile of hard-sphere fluids in a slit geometry can be evaluated to [119]

$$
n\left(x^{\perp} ; L\right)=\frac{n_{0}}{L}\left\{1+\pi\left(n_{0} L^{2}\right) g(\sigma)\left[\left(\frac{x^{\perp}}{L}\right)^{2}-\frac{1}{12}\right]+\mathcal{O}\left(n_{0} L^{2}\right)^{2}\right\},
$$

where the first-order correction is proportional to $g(\sigma)$, the 2D pair distribution function at contact. This factor also represents the curvature of the density profile. The density profile including the curvature is asymptotically an exact result, which quantifies the emergence of inhomogeneous packing in confined geometry. This analytical prediction is compared to hard-sphere simulations in Figure 12 for two different 2D packing fractions. One infers that the curvature of the density profile grows with increasing packing fraction. The comparison in Figure 12 for two moderate packing fractions indicates that the theoretical prediction is in fact an exact result in the limit of extreme confinement.

\subsubsection{Divergent coupling time}

The decoupling property in strong confinement suggests that in the limit $L \rightarrow 0$ the lateral and transversal degrees of freedom hardly influence each other. However, for small but finite $L$ the consequences of the weak coupling between lateral and transversal degrees of freedom on the dynamics have remained mostly unexplored.

For hard-sphere fluids the coupling between the transversal and lateral degrees of freedom occurs only via binary collisions. Yet, in extreme confinement the momentum transfer is almost planar, see Figure 10, and the coupling becomes weaker as the walls approach each other. Consequently, the binary collision operator may be replaced to 

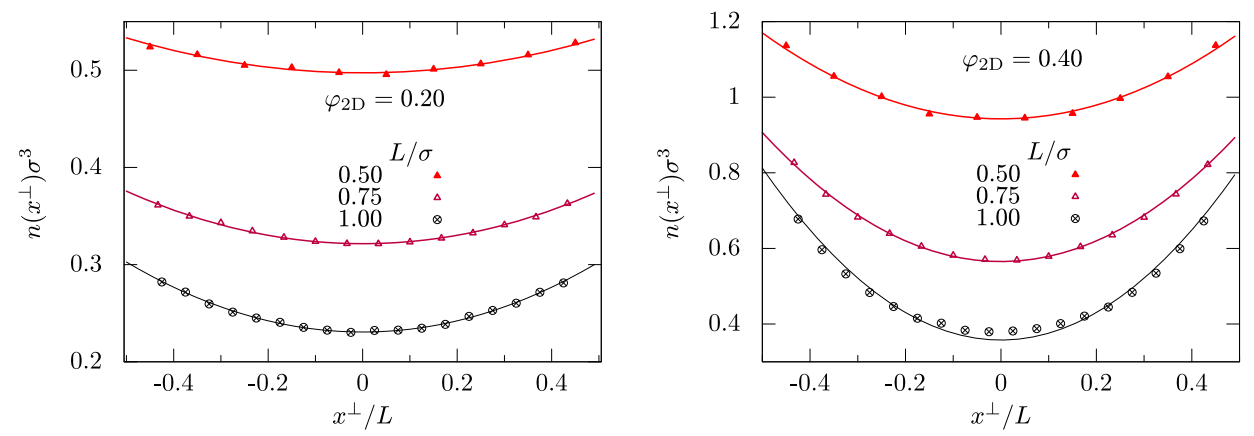

Fig. 12. The density profile in the direction perpendicular to the walls of quasi-2D hardsphere fluids for packing fractions $\varphi_{2 D}=0.20$ (left panel) and $\varphi_{2 D}=0.40$ (right panel), where $\varphi_{2 D}=(\pi / 4) \sigma^{2} n_{0}$ with number density $n_{0}$. The symbols are simulational results, whereas the dashed lines are the theoretical predictions given by equation (19).

leading order by its two-dimensional analog. The difference between the 3D and the $2 \mathrm{D}$ collision operator then corresponds to the small interaction between the lateral and transverse degrees of freedom. This weak coupling is expected to introduce a time scale up to which the coupling of the degrees of freedom is irrelevant. As the plate separation becomes smaller this time scale is expected to grow. The simplest way to identify this divergent time scale is to monitor a conserved quantity in the decoupled ensemble. In fact, the transversal kinetic energy of a tagged particle is a conserved quantity in the decoupled ensemble, however it will equilibrate slowly with the other degrees of freedom by the coupling. Employing the Zwanzig-Mori projection operator formalism $[1,67]$ an exact expression of the leading-order relaxation rate $\tau$ can be derived after performing the structural and kinetic averages [118]

$$
\tau^{-1}=\frac{16 \varphi_{2 D}}{3 \sqrt{\pi}}\left(\frac{L}{\sigma}\right)^{2} g(\sigma) t_{0}^{-1} .
$$

Here the scaling factor $(L / \sigma)^{2}$ reflects the small momentum transfer in quasi-planar collisions. This prediction has been confirmed by computer simulations only recently [118].

Although this analytical theory mainly deals with hard-core interactions, one anticipates that the results remain valid for the case of smooth potentials as long as the typical time for a particle to traverse the slit (Knudsen time scale) is still much larger than the duration of a collision. Then the mechanism for small transverse momentum transfer should be identical to the one for hard particles, see Figure 10.

It is also interesting to consider the opposite case where the duration of a collision is much longer than the time to traverse the slit. This can be realized by using relatively soft particles. Then the use of a collision operator is inappropriate, however the collision events can be averaged over the fast transverse oscillations. Analytic progress in this direction has been achieved [135] and it turns out that the predicted relaxation time diverges as $\sim L^{-3}$.

Here we test this prediction by tuning the softness of the interaction potential. Particle-particle interactions of the form $u_{p p}(r)=4 \epsilon\left[(\sigma / r)^{48}-(\sigma / r)^{24}\right]+\epsilon$ for $r \leq 2^{1 / 24} \sigma$ and $u_{p p}(r)=0$ for $r \geq 2{ }^{1 / 24} \sigma$ have been shown to mimic reliably hardsphere interactions $[136,137]$. For convenience, we employ the characteristic energy scale $\epsilon=k_{B} T$ which defines the time scale $t_{0}=\sqrt{m \sigma^{2} / \epsilon}$. Particles located inside 


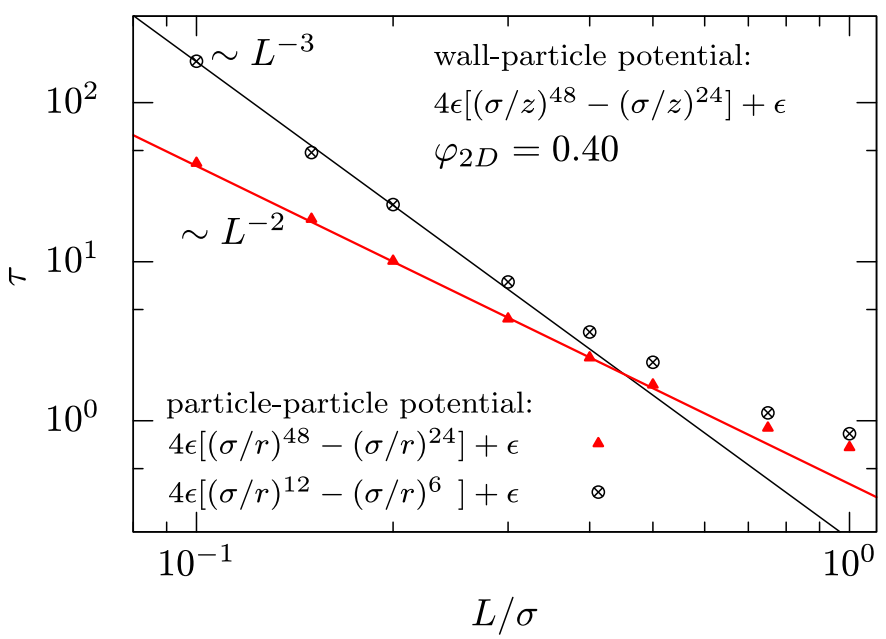

Fig. 13. Relaxation time $\tau$ for the dimensional crossover for strongly confined liquids as a function of the wall separation $L$ for two types of particle-particle interactions while the wall-particle interaction has been kept identical.

the slit interact with both walls, and we use the wall-particle interaction $u_{w p}(z)=$ $4 \epsilon\left[(\sigma / z)^{48}-(\sigma / z)^{24}\right]+\epsilon$ for $z \leq 2^{1 / 24} \sigma$ and $u_{w p}(z)=0$ for $z \geq 2^{1 / 24} \sigma$, where $z$ is the distance to one of the walls. The walls are located at $\pm(\sigma+L / 2)$, and the wallparticle interaction has been kept identical in all our simulations to realize hard walls. Figure 13 displays the dimensional crossover time for different particle-particle softnesses as the walls approach each other. One observes that the time scale divergence changes from $L^{-2}$ to $L^{-3}$ with increasing softness of the particles, which is in line with the above theoretical predictions. This implies that the conserved quantity in the decoupled ensemble of hard-core like particles will decay much faster than for soft particles.

\section{Summary}

Confinement has profound implications on the dynamics of tracers and dense liquids. The Lorentz model is a minimal model for the dynamics of tracers in disordered host structures which emerges naturally if a separation of time scales occurs. The dynamics in the vicinity of the localization threshold becomes anomalous and displays universal behavior as suggested from dynamical critical phenomena. In realistic systems some of the basic assumptions of the original Lorentz model need to be relaxed. While changing from uncorrelated obstacles to realistic quenched host matrices does not affect the universal properties, the way the matrix is probed dynamically is relevant. Furthermore, it would be interesting to investigate in detail how slowly rearranging matrices gradually change the transport properties.

For the case of the glass transition in confinement a striking multiple-reentrant scenario has been revealed both within mode-coupling theory and computer simulations. However, so far only the nonequilibrium state diagram and the nonergodicity parameters have been evaluated. Therefore there is a need for full dynamic solutions of the mode-coupling equations as well as for further computer simulations. Experiments in strong confinement appear to be feasible in colloidal systems provided one can avoid that particles stick to the wall. Interesting predictions that require further 
investigations concern the dimensional crossover from 2D to confined 3D systems, in particular, the possibility of a glass-glass transition deserves further attention.

We have benefited tremendously from collaboration and discussions over many years with Felix Höfling, Jürgen Horbach, Thomas Voigtmann, and Rolf Schilling. This work reviews the publications achieved within our project $[32,43,44,51,55,61,62,72,76,84,93,94,115-120$, $129,132-134]$ in the DFG research unit FOR1394, 'Nonlinear response to probe vitrification' for which we gratefully acknowledge financial support. Open access funding provided by Universität Innsbruck.

Open Access This is an Open Access article distributed under the terms of the Creative Commons Attribution License (http://creativecommons.org/licenses/by/4.0), which permits unrestricted use, distribution, and reproduction in any medium, provided the original work is properly cited.

\section{References}

1. W. Götze, Complex Dynamics of Glass-Forming Liquids: A Mode-Coupling Theory, International Series of Monographs on Physics (Oxford Science Publications, Oxford, 2009)

2. U. Bengtzelius, W. Götze, A. Sjolander, J. Phys. C: Solid State Phys. 17, 5915 (1984)

3. W. Götze, J. Phys.: Condens. Matter 11, A1 (1999)

4. W.M. Du, G. Li, H.Z. Cummins, M. Fuchs, J. Toulouse, L.A. Knauss, Phys. Rev. E 49, $2192(1994)$

5. H.Z. Cummins, G. Li, W.M. Du, J. Hernandez, N.J. Tao, Transp. Theory Statist. Phys. 24, 981 (1995)

6. H. Cummins, Y. Hwang, G. Li, W. Du, W. Losert, G. Shen, J. Non-Cryst. Solids 235, $254(1998)$

7. T. Franosch, W. Götze, M.R. Mayr, A.P. Singh, Phys. Rev. E 55, 3183 (1997)

8. A.P. Singh, G. Li, W. Götze, M. Fuchs, T. Franosch, H.Z. Cummins, J. Non-Cryst. Solids 235, 70 (1998)

9. W. Götze, Th. Voigtmann, Phys. Rev. E 61, 4133 (2000)

10. W. van Megen, S.M. Underwood, Phys. Rev. E 47, 248 (1993)

11. W. van Megen, T.C. Mortensen, S.R. Williams, J. Müller, Phys. Rev. E 58, 6073 (1998)

12. T. Franosch, M. Fuchs, W. Götze, M.R. Mayr, A.P. Singh, Phys. Rev. E 55, 7153 (1997)

13. M. Fuchs, W. Götze, M.R. Mayr, Phys. Rev. E 58, 3384 (1998)

14. W. Kob, H.C. Andersen, Phys. Rev. Lett. 73, 1376 (1994)

15. W. Kob, H.C. Andersen, Phys. Rev. E 51, 4626 (1995)

16. J. Bergenholtz, M. Fuchs, Phys. Rev. E 59, 5706 (1999)

17. K. Dawson, G. Foffi, M. Fuchs, W. Götze, F. Sciortino, M. Sperl, P. Tartaglia, Th. Voigtmann, E. Zaccarelli, Phys. Rev. E 63, 011401 (2000)

18. T. Eckert, E. Bartsch, Phys. Rev. Lett. 89, 125701 (2002)

19. K.N. Pham, A.M. Puertas, J. Bergenholtz, S.U. Egelhaaf, A. Moussaid, P.N. Pusey, A.B. Schofield, M.E. Cates, M. Fuchs, W.C.K. Poon, Science 296, 104 (2002)

20. K.N. Pham, S.U. Egelhaaf, P.N. Pusey, W.C.K. Poon, Phys. Rev. E 69, 011503 (2004)

21. A.M. Puertas, M. Fuchs, M.E. Cates, Phys. Rev. Lett. 88, 098301 (2002)

22. E. Zaccarelli, F. Sciortino, P. Tartaglia, J. Phys.: Condens. Matter 16, S4849 (2004)

23. W. Götze, M. Sperl, J. Phys.: Condens. Matter 15, S869 (2003)

24. M. Sperl, Phys. Rev. E 68, 031405 (2003)

25. W. Götze, M. Sperl, J. Phys.: Condens. Matter 16, S4807 (2004)

26. J. Horbach, W. Kob, K. Binder, Phys. Rev. Lett. 88, 125502 (2002)

27. J. Horbach, W. Kob, K. Binder, J. Phys.: Condens. Matter 15, S903 (2003) 
28. A. Meyer, J. Horbach, W. Kob, F. Kargl, H. Schober, Phys. Rev. Lett. 93, 027801 (2004)

29. F. Kargl, A. Meyer, M.M. Koza, H. Schober, Phys. Rev. B 74, 014304 (2006)

30. Th. Voigtmann, J. Horbach, Europhys. Lett. 74, 459 (2006)

31. A.J. Moreno, J. Colmenero, Phys. Rev. E 74, 021409 (2006)

32. J. Horbach, Th. Voigtmann, F. Höfling, T. Franosch, Eur. Phys. J. Special Topics 189, $141(2010)$

33. N. Kikuchi, J. Horbach, Europhys. Lett. 77, 26001 (2007)

34. A.J. Moreno, J. Colmenero, J. Phys.: Condens. Matter 19, 466112 (2007)

35. Th. Voigtmann, J. Horbach, Phys. Rev. Lett. 103, 205901 (2009)

36. T. Sentjabrskaja, E. Zaccarelli, C. De Michele, F. Sciortino, P. Tartaglia, Th. Voigtmann, S.U. Egelhaaf, M. Laurati, Nat. Commun. 7, 11133 (2016)

37. V. Krakoviack, Phys. Rev. Lett. 94, 065703 (2005)

38. V. Krakoviack, Phys. Rev. E 75, 031503 (2007)

39. V. Krakoviack, Phys. Rev. E 79, 061501 (2009)

40. V. Krakoviack, Phys. Rev. E 84, 050501 (2011)

41. W Götze, E. Leutheusser, S. Yip, Phys. Rev. A 23, 2634 (1981)

42. W. Götze, E. Leutheusser, S. Yip. Phys. Rev. A 25, 533 (1982)

43. S.K. Schnyder, F. Höfling, T. Franosch, Th. Voigtmann, J. Phys.: Condens. Matter 23, $234121(2011)$

44. S. Lang, V. Boţan, M. Oettel, D. Hajnal, T. Franosch, R. Schilling, Phys. Rev. Lett. 105, 125701 (2010)

45. H.A. Lorentz, Arch. Néerl. Sci. Exact Natur. 10, 336 (1905)

46. A. Cortis, B. Berkowitz, Soil Sci. Soc. Am. J. 68, 1539 (2004)

47. F. Höfling, T. Franosch, Rep. Prog. Phys. 76, 046602 (2013)

48. R.J. Ellis, Trends in Biochem. Sci. 26, 597 (2001)

49. D. Hall, A.P. Minton, BBA-Proteins Proteom 1649, 127 (2003)

50. M. Saxton, Biophys. J. 66, 394 (1994)

51. T. Bauer, F. Höfling, T. Munk, E. Frey, T. Franosch, Eur. Phys. J. Special Topics 189, $103(2010)$

52. J. Kertész, J. Phys. (Paris) 42, 393 (1981)

53. W.T. Elam, A.R. Kerstein, J.J. Rehr, Phys. Rev. Lett. 52, 1516 (1984)

54. F. Höfling, T. Franosch, E. Frey, Phys. Rev. Lett. 96, 165901 (2006)

55. M. Spanner, F. Höfling, S.C. Kapfer, K.R. Mecke, G.E. Schröder-Turk, T. Franosch, Phys. Rev. Lett. 116, 060601 (2016)

56. A.R. Kerstein, J. Phys. A 16, 3071 (1983)

57. C.H. Rycroft, Chaos 19, 041111 (2009)

58. P.G. de Gennes, La Recherche 7, 919 (1976)

59. D. ben Avraham, S. Havlin, Diffusion and Reactions in Fractals and Disordered Systems (Cambridge University Press, Cambridge, 2000)

60. S. Havlin, D. Ben-Avraham, Adv. Phys. 51, 187 (2002)

61. T. Franosch, M. Spanner, T. Bauer, G.E. Schröder-Turk, F. Höfling, J. Non-Cryst. Solids 357, 472 (2011)

62. M. Spanner, F. Höfling, G.E. Schröder-Turk, K. Mecke, T. Franosch, J. Phys.: Condens. Matter 23, 234120 (2011)

63. J. Machta, R.A. Guyer, S.M. Moore, Phys. Rev. B 334818 (1986)

64. A. Kammerer, F. Höfling, T. Franosch, Europhys. Lett. 84, 66002 (2008)

65. F. Höfling, T. Munk, E. Frey, T. Franosch, J. Chem. Phys. 128, 164517 (2008)

66. J. Kertész, J. Metzger, J. Phys. A 16, L735 (1983)

67. J.P. Hansen, I.R. McDonald, Theory of Simple Liquids (Academic Press, 2006)

68. R. Cerbino, V. Trappe, Phys. Rev. Lett. 100, 188102 (2008)

69. P.J. Lu, F. Giavazzi, T.E. Angelini, E. Zaccarelli, F. Jargstorff, A.B. Schofield, J.N. Wilking, M.B. Romanowsky, D.A. Weitz, R. Cerbino, Phys. Rev. Lett. 108, 218103 (2012) 
70. L.G. Wilson, V.A. Martinez, J. Schwarz-Linek, J. Tailleur, G. Bryant, P.N. Pusey, W.C.K. Poon, Phys. Rev. Lett. 106, 018101 (2011)

71. C. Kurzthaler, S. Leitmann, T. Franosch, Sci. Rep. 6, 36702 (2016)

72. M. Spanner, S.K. Schnyder, F. Höfling, Th. Voigtmann, T. Franosch, Soft Matter 9, $1604(2013)$

73. F. Höfling, K.-U. Bamberg, T. Franosch, Soft Matter 7, 1358 (2011)

74. T.O.E. Skinner, S.K. Schnyder, D.G.A.L. Aarts, J. Horbach, R.P.A. Dullens, Phys. Rev. Lett. 111, 128301 (2013)

75. A.L. Thorneywork, R.E. Rozas, R.P.A. Dullens, J. Horbach, Phys. Rev. Lett. 115, $268301(2015)$

76. S.K. Schnyder, M. Spanner, F. Höfling, T. Franosch, J. Horbach, Soft Matter 11, 701 (2015)

77. F. Höfling (unpublished)

78. P.C. Hohenberg, B.I. Halperin, Rev. Mod. Phys. 49, 435 (1977)

79. R. Folk, G. Moser, J. Phys. A 39, R207 (2006)

80. J.P. Straley, J. Phys. C 15, 2343 (1982)

81. F. Höfling, T. Franosch, Phys. Rev. Lett. 98, 140601 (2007)

82. B.I. Halperin, S. Feng, P.N. Sen, Phys. Rev. Lett. 54, 2391 (1985)

83. A. Kuzmany, H. Spohn, Phys. Rev. E 57, 5544 (1998)

84. W. Schirmacher, B. Fuchs, F. Höfling, T. Franosch, Phys. Rev. Lett. 115, 240602 (2015)

85. A. Weijland, J.M.J. van Leeuwen, Physica (Amsterdam) 38, 35 (1968)

86. H. van Beijeren, Rev. Mod. Phys. 54, 195 (1982)

87. M.H. Ernst, A. Weijland, Phys. Lett. A 34, 39 (1971)

88. T. Franosch, F. Höfling, T. Bauer, E. Frey, Chem. Phys. 375, 540 (2010)

89. Th.M. Nieuwenhuizen, P.F.J. van Velthoven, M.H. Ernst, J. Phys. A: Math. Gen. 20, 4001 (1987)

90. D. Frenkel, Phys. Lett. A 121, 385 (1987)

91. M.H. Ernst, G.A. van Velzen, J. Stat. Phys. 57, 455 (1989)

92. D. Frenkel, F. van Luijn, P.-M. Binder, Europhys. Lett. 20, 7 (1992)

93. S. Leitmann, T. Franosch, Phys. Rev. Lett. 111, 190603 (2013)

94. S. Leitmann, T. Franosch, Phys. Rev. Lett. 118, 018001 (2017)

95. U. Basu, C. Maes, J. Phys. A 47, 255003 (2014)

96. O. Bénichou, P. Illien, G. Oshanin, A. Sarracino, R. Voituriez, Phys. Rev. E 93, 032128 (2016)

97. Th. M. Nieuwenhuizen, P.F.J. van Velthoven, M.H. Ernst, Phys. Rev. Lett. 57, 2477 (1986)

98. Th. M. Nieuwenhuizen, P.F.J. van Velthoven, M.H. Ernst, J. Phys. A 20, 4001 (1987)

99. M.H. Ernst, Th. M. Nieuwenhuizen, P.F.J. van Velthoven, J. Phys. A 20, 5335 (1987)

100. R.N. Zia, J.F. Brady, J. Fluid Mech. 658, 9 (2010)

101. O. Bénichou, A. Bodrova, D. Chakraborty, P. Illien, A. Law, C. Mejía-Monasterio, G. Oshanin, R. Voituriez, Phys. Rev. Lett. 111, 260601 (2013)

102. V. Dmery, O. Bnichou, H. Jacquin, New J. Phys. 16, 053032 (2014)

103. T.M. Squires, J.F. Brady, Phys. Fluids 17, 073101 (2005)

104. P. Scheidler, W. Kob, K. Binder, J. Phys. IV 10, 33 (2000)

105. J. Mittal, T.M. Truskett, J.R. Errington, G. Hummer, Phys. Rev. Lett. 100, 145901 (2008)

106. C.R. Nugent, K.V. Edmond, H.N. Patel, E.R. Weeks, Phys. Rev. Lett. 99, 025702 (2007)

107. K. Nygård, R. Kjellander, S. Sarman, S. Chodankar, E. Perret, J. Buitenhuis, J.F. van der Veen, Phys. Rev. Lett. 108, 037802 (2012)

108. J. Baschnagel, F. Varnik, J. Phys. Condens. Matter 17, R851 (2005)

109. W.P. Krekelberg, V.K. Shen, J.R. Errington, T.M. Truskett, J. Chem. Phys. 135, $154502(2011)$

110. J. Mittal, J.R. Errington, T.M. Truskett, Phys. Rev. Lett. 96, 177804 (2006) 
111. T.S. Ingebrigtsen, J.R. Errington, T.M. Truskett, J.C. Dyre, Phys. Rev. Lett. 111 235901 (2013)

112. G. Foffi, W. Götze, F. Sciortino, P. Tartaglia, Th. Voigtmann, Phys. Rev. Lett. 91, $085701(2003)$

113. E. Zaccarelli, H. Löwen, P.P.F. Wessels, F. Sciortino, P. Tartaglia, C.N. Likos, Phys. Rev. Lett. 92, 225703 (2004)

114. S. Lang, R. Schilling, V. Krakoviack, T. Franosch, Phys. Rev. E 86, 021502, (2012)

115. S. Mandal, S. Lang, M. Gross, M. Oettel, D. Raabe, T. Franosch, F. Varnik, Nat. Commun. 5, 4435 (2014)

116. T. Franosch, J. Phys. A: Math. Theor. 47, 325004 (2014)

117. F. Varnik, T. Franosch, J. Phys.: Condens. Matter 28, 133001 (2016)

118. S. Mandal, T. Franosch, Phys. Rev. Lett. 118, 065901 (2017)

119. S. Lang, R. Schilling, T. Franosch, Phys. Rev. E 90, 062126 (2014)

120. S. Lang, T. Franosch, Phys. Rev. E 89, 062122 (2014)

121. R. Haussmann, Z. Phys. B 79, 143 (1990)

122. W. Götze, L. Sjögren, J. Math. Anal. Appl. 195, 230 (1995)

123. T. Franosch, Th. Voigtmann, J. Stat. Phys. 109, 237 (2002)

124. S. Lang, R. Schilling, T. Franosch, J. Stat. Mech. Theor. Exp. 2013, P12007 (2013)

125. R. Schilling, T. Scheidsteger, Phys. Rev. E 56, 2932 (1997)

126. T. Franosch, M. Fuchs, W. Götze, M.R. Mayr, A.P. Singh, Phys. Rev. E 56, 5659 (1997)

127. K. Nygård, S. Sarman, R. Kjellander, J. Chem. Phys. 139, 164701 (2013)

128. D. Henderson, Fundamentals of Inhomogeneous Fluids (Dekker, New York, 1992)

129. S. Mandal, T. Franosch (submitted, 2017)

130. H. Löwen, J. Phys.: Condens. Matter 13, R415 (2001)

131. A. Fortini, M. Dijkstra, J. Phys.: Condens. Matter 18, L371 (2006)

132. T. Franosch, S. Lang, R. Schilling, Phys. Rev. Lett. 109, 240601 (2012)

133. T. Franosch, S. Lang, R. Schilling, Phys. Rev. Lett. 110, 059901 (2013)

134. S. Lang, T. Franosch, R. Schilling, J. Chem. Phys. 140, 104506 (2014)

135. R. Schilling, Phys. Rev. E 93, 062102 (2016)

136. J.A. Bollinger, A. Jain, J. Carmer, T.M. Truskett, J. Chem. Phys. 142, 161102 (2015)

137. J.A. Bollinger, J. Carmer, A. Jain, T.M. Truskett, Soft Matter 12, 9561 (2016) 